\title{
Finding a Stable Matching under Type-specific Minimum Quotas*
}

\author{
Kentaro Tomoeda ${ }^{\dagger}$
}

March 17, 2018

\begin{abstract}
In matching problems with minimum and maximum type-specific quotas, there may not exist a stable (i.e., fair and non-wasteful) assignment (Ehlers et al., 2014). This paper investigates the structure of schools' priority rankings which guarantees stability. First, we show that there always exists a fair and non-wasteful assignment if for each type of students, schools have common priority rankings over a certain number of bottom students. Next, we show that the pairwise version of this condition characterizes the maximal domain of two schools' priority rankings over same type students to guarantee the existence of stable assignments. To prove the existence theorem, we propose a new mechanism Deferred Acceptance with Precedence Lists (DAPL), which is feasible, non-wasteful, strictly PL-fair and group strategy-proof for any priority rankings. Strict PL-fairness is weaker than fairness, but DAPL satisfies fairness under our sufficient condition. We also show that there is no strategy-proof mechanism that Pareto dominates DAPL whenever the outcome of DAPL is Pareto dominated by a stable assignment.
\end{abstract}

${ }^{*}$ This is originally Chapter 3 of my Ph.D. thesis at Harvard University. I am very grateful to my advisors Eric Maskin, Scott Kominers and Edward Glaeser for their constant guidance. I am grateful to Lars Ehlers, Isa Hafalir, Ming He, Daisuke Hirata, Kenzo Imamura, Yusuke Kasuya, Alvin Roth, Emil Temnyalov, Peter Troyan, Bumin Yenmez, Jun Zhang, Mengxi Zhang, and Xin Zhao for helpful discussions. Seminar participants at GRIPS Market Design Conference, University of Technology Sydney, University of Queensland, Asian Meeting of Econometric Society 2017, Kyoto University, University of New South Wales, 11th Japan-Taiwan-Hong Kong Contract Theory Conference, Asia-Pacific Industrial Organisation Conference 2017 and Australasian Economic Theory Workshop 2018 provided useful comments. I also thank the editor, the associate editor and three referees for helpful comments and suggestions.

$\dagger$ University of Technology Sydney, UTS Business School, PO Box 123, Broadway NSW 2007, Australia, Kentaro.Tomoeda@uts.edu.au. 
Keywords: type-specific minimum quotas, stability, priority rankings, deferred acceptance, controlled school choice

JEL Classification Numbers: C78, D47, D82

\section{Introduction}

While the modern matching theory has provided solutions to many real-world allocation problems, there are still important problems that have yet to be solved due to technical difficulties. One of such problems is matching with type-specific minimum quotas. In many school districts in the United States, maximum and minimum quotas are separately set for different groups of students in order to achieve gender, racial or socioeconomic diversity at schools. For instance, New York City requires Educational Option (EdOpt) schools to keep the diversity of ability levels by accepting students from different ranges of test scores. For each EdOpt school, 16 percent of students must score below the grade level on the standardized test, 68 percent must score at the grade level, and 16 percent must score above the grade level (Abdulkadiroğlu et al., 2005). In the public school choice program in Cambridge, US, students are classified into high and low socioeconomic groups, and each school is required to admit certain percentages of students from each group (Fragiadakis and Troyan, 2017). Type-specific minimum quotas are also observed in the matching problems between students and supervisors (Kawagoe and Matsubae, 2017). University departments (especially in natural science) set minimum quotas to supervisors because (i) supervisors need some students to operate their laboratories, and (ii) the departments aim to achieve an equal share of educational burden among the supervisors. And in many cases, these constraints only apply to students from a certain program or field, which requires quotas be type-specific.

Despite the prevalence of type-specific minimum quotas, theoretically proposing a desirable solution to this problem is still a difficult task. Ehlers (2010) and Ehlers et al. (2014) formulated this problem and found a general impossibility result that the set of feasible, fair and non-wasteful assignments may be empty (Theorem 1). This is in contrast with the standard matching problem with only maximum type-specific quotas, where we can always find a stable assignment by the Deferred Acceptance (DA) mechanism (Abdulkadiroğlu and Sönmez, 2003; Abdulkadiroğlu, 2005). And in most of the literature which pursued stability in this problem, the authors proposed solutions by (i) interpreting constraints as soft bounds (Ehlers et al., 2014) or (ii) dropping or weakening one of (or both of) fairness and non-wastefulness (Ehlers et al., 2014; Fragiadakis and Troyan, 2017; Goto et al., 2016, 2017). 
In this paper, we propose designing the priority rankings of schools to ensure stability without weakening stability itself. ${ }^{1,2}$ As we discuss in subsection 3.1 and Section 4, the priority structure is part of the choice variables for the mechanism designer in many applications. To design priority rankings appropriately, we need to answer the following question: What is the domain of priority rankings for which we can (or cannot) ensure the existence of fair and non-wasteful assignments under type-specific constraints? We first provide a positive answer to this. When schools have common priority among a certain number of bottom students for each type (we call this condition B-common priority), we show that there always exists a feasible, fair and non-wasteful assignment (Theorem 2). The threshold of bottom students varies across schools, and it is computed by the capacity and type-specific constraints of all schools. Intuitively, if a student is ranked lower than other same type students at some school (and if her ranking is low enough), then she is so in any other school as well. The priority order between different types of students can differ across schools in an arbitrary way.

Second, we also show that the pairwise version of B-common priority characterizes the maximal domain of two schools' priority rankings over same type students to guarantee the existence of a feasible, fair and non-wasteful assignment (Theorem 3). This implies that pairwise B-common priority is necessary (in a weak sense) to ensure stability.

To show the existence theorem, we propose a new mechanism called Deferred Acceptance Mechanism with Precedence Lists (DAPL). In DAPL, we transform the original problem into a corresponding problem with only maximum quotas. To be more specific, we divide the schools into smaller divisions, and add a null division for each type of students. If students are assigned to null divisions at the end of the DA stage, they must fill the remaining minimum quotas. In order to successfully sort students into the school divisions and the null divisions using the standard DA, we add a certain number of artificial students. The priority order of each null division is defined by an exogenously given precedence list.

DAPL has many desirable properties for general priority rankings of schools. We show that DAPL is always (i) feasible, (ii) non-wasteful, (iii) strictly PL-fair, and (iv) group strategy-proof (Proposition 1). Although strict PL-fairness is a stronger version of PLfairness introduced by Fragiadakis et al. (2015), it does not preclude a certain class of justified envies, and hence it is weaker than the standard fairness. In Proposition 2, we

\footnotetext{
${ }^{1}$ We employ the most natural definition of stability proposed by Ehlers et al. (2014) in our model.

${ }^{2}$ In the literature, the domain of schools' priority rankings is studied to understand the relationship among several properties such as efficiency, stability and strategy-proofness for school choice mechanisms (Ergin, 2002; Kesten, 2006; Haeringer and Klijn, 2009; Kumano, 2013).
} 
show that DAPL is also fair for problems with B-common priority. The outcome of DAPL is not Pareto dominated by a feasible, fair and non-wasteful assignment for problems with only one type (Proposition 3), but it is not the case when there are more than one types (Observation 1). In general, we can show that unless we give up strategy-proofness, no mechanism can Pareto improve DAPL whenever DAPL is Pareto dominated by a feasible, fair and non-wasteful assignment (Proposition 4).

In the literature of matching theory, a growing number of papers study complex constraints and affirmative action in matching markets. ${ }^{3}$ But to the best of our knowledge, we are the first one to provide conditions for the existence of fair and non-wasteful assignments under type-specific minimum quotas. Moreover, our existence result cannot be proved by other existing mechanisms.

Among others, the following papers are most relevant to our work. Ehlers et al. (2014) weakened both the fairness and the non-wastefulness conditions, and showed that there exists an assignment that is fair for same types and constrained non-wasteful. ${ }^{4}$ They also proposed the soft-bound interpretation of constraints, and showed that the student-proposing DA mechanism finds an assignment that minimizes violations of controlled choice constraints among fair assignments. Fragiadakis and Troyan (2017) introduced a new dynamic quotas mechanism, called DQDA, which improves upon the benchmark DA mechanism while satisfying fairness for same types and strategy-proofness. But depending on the choice of the reduction sequence, DQDA may be wasteful under the common priority condition. Goto et al. (2017) propose a mechanism called ADA, which satisfies strategy-proofness, nonwastefulness and a weaker fairness property under general distributional constraints. They also show that ADA can be applied to our problem, but ADA may not be fair across different types even under common priority. Kawagoe and Matsubae (2017) report a real-world application of this problem in Future University Hakodate. We will carefully examine their student-supervisor matching problem in Section 4.

Minimum quotas are introduced to matching problems in many other ways. Fragiadakis et al. (2015), Goto et al. (2016) and Kurata et al. (2016) consider minimum quotas, but

\footnotetext{
${ }^{3}$ Recent works on affirmative action and diversity in matching problems include Biró et al. (2010), Erdil and Kumano (2012), Kojima (2012), Hafalir et al. (2013), Westkamp (2013), Echenique and Yenmez (2015), Aygün and Bó (2016), Aygün and Turhan (2016), Bó (2016), Doğan (2016), Kominers and Sönmez (2016), Dur et al. (2017) and Dur et al. (forthcoming). For other forms of important distributional constraints in matching markets, see Kamada and Kojima (2015, 2017, forthcoming) and Delacrétaz et al. (2016).

${ }^{4} \mathrm{An}$ assignment is constrained non-wasteful if, when a student justifiably claims an empty slot at a school, the resulting assignment in which the student is assigned to that school is not fair for same types. See Section 2 for the definitions of non-wastefulness and fairness.
} 
their models do not allow schools to have a separate floor for each type of students. The multistage DA mechanism (MSDA) proposed by Fragiadakis et al. (2015) has a similar idea with our DAPL in the sense that the precedence lists determine which students are allowed to propose to schools. But in problems with one type of students, MSDA does not necessarily find a fair and non-wasteful assignment under the B-common priority condition. We provide an example to illustrate this in subsection 3.4. Hafalir et al. (2017) consider a matching problem where multiple school districts are involved, and analyze type-specific minimum quotas imposed on each district, not on each school. When they consider these minimum quotas, they focus on strategy-proofness and efficiency rather than stability.

The rest of the paper proceeds as follows. Section 2 introduces the model. Section 3 presents all the results. In subsection 3.1, we define the B-common priority condition, and in subsection 3.2, we introduce DAPL and show the main properties of DAPL. Our existence theorem is proved by using DAPL. We examine the efficiency of DAPL in subsection 3.3, and compare DAPL and MSDA by Fragiadakis et al. (2015) in subsection 3.4. The maximal domain theorem is in subsection 3.5. We discuss the student-supervisor matching problem as an application in Section 4, and Section 5 concludes. All lemmas and proofs are in the Appendix.

\section{Model}

We consider a school choice problem with type-specific maximum and minimum quotas. This is also called the controlled school choice problem. Our model consists of the following elements:

1. a finite set of students $S=\left\{s_{1}, \ldots, s_{n}\right\}$;

2. a finite set of schools $C=\left\{c_{1}, \ldots, c_{m}\right\}$ where $m \geq 2$;

3. a capacity vector $q=\left(q_{c_{1}}, \ldots, q_{c_{m}}\right)$, where $q_{c}$ is the capacity of school $c \in C$ or the number of seats in $c \in C$ such that $n \leq \sum_{c \in C} q_{c}$;

4. a preference profile of students $P_{S}=\left(P_{s_{1}}, \ldots, P_{s_{n}}\right)$, where $P_{s}$ is the strict preference relation of student $s \in S$ over $C$, i.e. $c P_{s} c^{\prime}$ means that student $s$ strictly prefers school $c$ to school $c^{\prime}$

5. a priority profile of schools $\succ_{C}=\left(\succ_{c_{1}}, \ldots, \succ_{c_{m}}\right)$, where $\succ_{c}$ is the strict priority ranking of school $c \in C$ over $S$; $s \succ_{c} s^{\prime}$ means that student $s$ has higher priority than student $s^{\prime}$ to be enrolled at school $c$; 
6. a type space $T=\left\{t_{1}, \ldots, t_{k}\right\}$;

7. a type function $\tau: S \rightarrow T$, where $\tau(s)$ is the type of student $s$;

8. for each school $c$, two vectors of type-specific constraints $\underline{q}_{c}^{T}=\left(\underline{q}_{c}^{t_{1}}, \ldots, \underline{q}_{c}^{t_{k}}\right)$ and $\bar{q}_{c}^{T}=$ $\left(\bar{q}_{c}^{t_{1}}, \ldots, \bar{q}_{c}^{t_{k}}\right)$ such that $\underline{q}_{c}^{t} \leq \bar{q}_{c}^{t} \leq q_{c}$ for all $t \in T$, and $\sum_{t \in T} \underline{q}_{c}^{t} \leq q_{c} \leq \sum_{t \in T} \bar{q}_{c}^{t} .{ }^{5}$

We call $\left(S, C,\left(q_{c}\right)_{c \in C}, T, \tau,\left(\underline{q}_{c}^{T}, \bar{q}_{c}^{T}\right)_{c \in C}\right) \equiv \mathcal{Z}$ a primitive problem, and $\left(\mathcal{Z}, \succ_{C}\right)$ a problem.

Note that we assume all schools and students are acceptable to each other. ${ }^{6} \underline{q}_{c}^{t}$ is the minimal number of slots that school $c$ must allocate to students of type $t$ and $\bar{q}_{c}^{t}$ is the maximal number of slots that school $c$ is allowed to allocate to students of type $t$. We call $\left(\underline{q}_{c}^{t}, \bar{q}_{c}^{t}\right)$ the floor and the ceiling for type $t$ at school $c$. For each type $t \in T$, let $S_{t}$ be the set of type- $t$ students, i.e., $S_{t} \equiv\{s \in S \mid \tau(s)=t\}$.

The first five elements consist of a standard school choice problem considered by Abdulkadiroğlu and Sönmez (2003). They also introduced types of students as an extension and considered type-specific maximum quotas. In a separate paper, Abdulkadiroğlu (2005) considers the college admission model with type-specific quotas. The difference between our model and theirs is that we have minimum quotas for each type of students in addition to maximum quotas. As we see in the following sections, the discussions based on the standard DA mechanism in Abdulkadiroğlu and Sönmez (2003) and Abdulkadiroğlu (2005) no longer work when we have minimum quotas.

An assignment $\mu$ is a function from $C \cup S$ to $C \cup 2^{S}$ such that

1. $\mu(s) \in C$ for every student $s \in S$;

2. $|\mu(c)| \leq q_{c}$ and $\mu(c) \subseteq S$ for every school $c \in C$;

3. $\mu(s)=c$ if and only if $s \in \mu(c)$.

$\mu(s)$ denotes the school that student $s$ is assigned to, and $\mu(c)$ denotes the set of students who are assigned to school $c$. Under an assignment, every student is assigned to a school, and every school $c$ can accept at most $q_{c}$ students. Let $\mu^{t}(c) \equiv \mu(c) \cap S_{t}$ denote the set of type- $t$ students who are assigned to school $c$ under $\mu$. An assignment $\mu$ is feasible if $\underline{q}_{c}^{t} \leq\left|\mu^{t}(c)\right| \leq \bar{q}_{c}^{t}$ holds for every $(t, c) \in T \times C$. Let $F$ be the set of all feasible assignments.

\footnotetext{
${ }^{5} q_{c} \leq \sum_{t \in T} \bar{q}_{c}^{t}$ is without loss of generality because we cannot assign more students than $\sum_{t \in T} \bar{q}_{c}^{t}$ to school $c$ even if $q_{c}$ is strictly larger than that.

${ }^{6}$ In many matching problems without minimum quotas, schools may be unacceptable to students. But if students prefer their outside options to some schools, we may not be able to fill minimum quotas without violating individual rationality. Thus, here we assume that every school is acceptable to every student.
} 
A controlled school choice problem may not have a feasible solution because of the typespecific constraints. For example, we cannot find any feasible assignment if the sum of all floors (ceilings) for a certain type is greater (less) than the number of students of that type. To avoid such cases, we only consider constraints such that a feasible assignment exists. We also assume $\sum_{c \in C} \underline{q}_{c}^{t}<\left|S_{t}\right|<\sum_{c \in C} \bar{q}_{c}^{t}$ for each type $t \in T$. This is because if one of the two inequalities is equal, the number of feasible type- $t$ slots at each school is uniquely determined, and the standard DA mechanism could independently assign type- $t$ students to these slots in a fair manner.

As we show in subsection 3.1, this feasibility requirement leads to impossibility results: there may not exist a feasible assignment that is fair and non-wasteful. Given these results, Ehlers et al. (2014) propose type-specific constraints to be interpreted as soft bounds: schools can admit fewer students than their floors or more students than their ceilings, but they give highest priority to types who do not fill their floors, medium priority to types who fill their floors but not ceilings, and lowest priorities to types who fill their ceilings. In many applications, however, the goal of the designer is to achieve a desirable distribution of students rather than just to give high priority to a certain type of students. Thus, in this paper, we stick to the hard-bound interpretation and focus on the domain of schools' priority rankings. Hereafter, when we just say an "assignment," we implicitly mean that it is a feasible assignment.

Next, let us introduce the definition of stability in this problem. We follow Ehlers et al. (2014) and consider natural adaptations of non-wastefulness and fairness from the standard two-sided matching literature (without type-specific constraints). More specifically, these definitions take account of our feasibility requirements when justifying claims of empty slots and envies.

The non-wastefulness condition considered by Balinski and Sönmez (1999) is defined in our problem in the following way.

Definition 1. Student s justifiably claims an empty slot at school $c$ under a feasible assignment $\mu$ if

$$
\begin{aligned}
& \text { (nw1) } c P_{s} \mu(s) \text { and }|\mu(c)|<q_{c}, \\
& (\mathrm{nw} 2) \underline{q}_{\mu(s)}^{\tau(s)}<\left|\mu^{\tau(s)}(\mu(s))\right|, \text { and } \\
& (\mathrm{nw} 3)\left|\mu^{\tau(s)}(c)\right|<\bar{q}_{c}^{\tau(s)} .
\end{aligned}
$$

A feasible assignment $\mu$ is non-wasteful if there is no student who justifiably claims an empty slot at any school. 
(nw1) means that student $s$ prefers an empty slot at school $c$ to her assigned school $\mu(s)$. (nw2) means that the floor of student s's type at school $\mu(s)$ is not binding, and (nw3) means that the ceiling of student $s$ 's type at school $c$ is not binding either. In short, these three conditions imply that the constraints will not be violated when $s$ is assigned her more preferable empty slot at $c$ without changing other students' assignments.

As in the case of non-wastefulness, envy is justified only when student $s$ prefers some school to her own assignment, there is another student $s^{\prime}$ who has lower priority in the school, and replacing $s^{\prime}$ with $s$ would not violate the constraints. Since the mathematical representation depends on whether $s$ and $s^{\prime}$ are of the same type or not, we define envy for same type students and for students of different types separately.

Definition 2 formally states the conditions for a student to justifiably envy students of different types.

Definition 2. Student s justifiably envies student $s^{\prime}$ of a different type at school $c$ under an assignment $\mu$ if there exists another feasible assignment $\mu^{\prime}$ such that

(f1) $\mu\left(s^{\prime}\right)=c, c P_{s} \mu(s)$ and $s \succ_{c} s^{\prime}$,

(f2) $\mu^{\prime}(s)=c, \mu^{\prime}\left(s^{\prime}\right) \neq c$ and $\mu^{\prime}(\hat{s})=\mu(\hat{s})$ for all $\hat{s} \in S \backslash\left\{s, s^{\prime}\right\}$, and

(f3) $\tau(s) \neq \tau\left(s^{\prime}\right)$.

A feasible assignment $\mu$ is fair across different types if there is no student who justifiably envies another student of a different type.

(f1) is the standard condition that student $s$ prefers school $c$ to her assigned school $\mu(s)$, but there is a student $s^{\prime}$ who is assigned to school $c$ and has lower priority than $s$ at school $c$. (f2) means that assigning student $s$ a slot at school $c$, student $s^{\prime}$ a slot at some school other than $c$, and keeping all the other assignments unchanged do not violate the constraints at any school. (f3) says that $s$ and $s^{\prime}$ are of different types.

Between same type students, justified envy is defined in a simpler way. This is because feasibility will not be violated when two students of the same type exchange their slots.

Definition 3. Student s justifiably envies student $s^{\prime}$ of the same type at school c under an assignment $\mu$ if

$$
\begin{aligned}
& \left(\mathrm{f} 1^{*}\right) \mu\left(s^{\prime}\right)=c, c P_{s} \mu(s), s \succ_{c} s^{\prime}, \text { and } \\
& \left(\mathfrak{f} 2^{*}\right) \tau(s)=\tau\left(s^{\prime}\right)
\end{aligned}
$$


A feasible assignment $\mu$ is fair for same types if there is no student who justifiably envies any other same type student.

$\left(\mathrm{f} 1^{*}\right)$ is the same condition as (f1). Since student $s$ and $s^{\prime}$ are of the same type by (f2*), the assignment is still feasible when they exchange their slots. Namely, we can construct a feasible assignment $\mu^{\prime}$ in the following way: $\mu^{\prime}(s)=\mu\left(s^{\prime}\right), \mu^{\prime}\left(s^{\prime}\right)=\mu(s)$, and $\mu^{\prime}\left(s^{\prime \prime}\right)=\mu\left(s^{\prime \prime}\right)$ for all $s^{\prime \prime} \in S \backslash\left\{s, s^{\prime}\right\}$. We say that a feasible assignment $\mu$ is fair if it is both fair across different types and fair for same types. We also use "stability" to mean both non-wastefulness and fairness.

A mechanism $\gamma$ is a function from the set of all problems and student preferences to the set of all assignments of these problems. We write the assignment of $s \in S$ under $\gamma\left(\mathcal{Z}, \succ_{C}, P_{S}\right)$ by $\gamma_{s}\left(\mathcal{Z}, \succ_{C}, P_{S}\right)$. We say that a mechanism $\gamma$ satisfies property $X$ if $\gamma\left(\mathcal{Z}, \succ_{C}, P_{S}\right)$ satisfies property $X$ for any $\left(\mathcal{Z}, \succ_{C}, P_{S}\right)$. We also say that a mechanism $\gamma$ satisfies property $X$ for problem $\left(\mathcal{Z}, \succ_{C}\right)$ if $\gamma\left(\mathcal{Z}, \succ_{C}, P_{S}\right)$ satisfies property $X$ for any $P_{S}$. A mechanism $\gamma$ is strategy-proof if it is a dominant strategy for each student to truthfully report her preference in any problem, i.e., for any problem $\left(\mathcal{Z}, \succ_{C}\right)$ and profile of student preferences $P_{S}$, there is no student $s \in S$ and her preference $P_{s}^{\prime}$ such that $\gamma_{s}\left(\mathcal{Z}, \succ_{C}, P_{s}^{\prime}, P_{-s}\right) P_{s} \gamma_{s}\left(\mathcal{Z}, \succ_{C}, P_{S}\right)$. A mechanism $\gamma$ is group strategy-proof if for any problem $\left(\mathcal{Z}, \succ_{C}\right)$ and profile of student preferences $P_{S}$, there is no group of students $I \subseteq S$ and their preference profile $P_{I}^{\prime}$ such that $\gamma_{s}\left(\mathcal{Z}, \succ_{C}, P_{I}^{\prime}, P_{S \backslash I}\right) P_{s} \gamma_{s}\left(\mathcal{Z}, \succ_{C}, P_{S}\right)$ for all $s \in I$.

An assignment $\mu$ weakly Pareto dominates $\mu^{\prime}$ if $\mu(s) P_{s} \mu^{\prime}(s)$ or $\mu(s)=\mu^{\prime}(s)$ for any $s \in S$. An assignment $\mu$ Pareto dominates $\mu^{\prime}$ if $\mu$ weakly Pareto dominates $\mu^{\prime}$, and $\mu(s) P_{s} \mu^{\prime}(s)$ for some $s \in S$. An assignment $\mu$ is Pareto efficient if $\mu$ is not Pareto dominated by any other feasible assignment. A mechanism $\gamma$ weakly Pareto dominates $\gamma^{\prime}$ if $\gamma\left(\mathcal{Z}, \succ_{C}, P_{S}\right)$ weakly Pareto dominates $\gamma^{\prime}\left(\mathcal{Z}, \succ_{C}, P_{S}\right)$ for any $\left(\mathcal{Z}, \succ_{C}, P_{S}\right)$. A mechanism $\gamma$ Pareto dominates $\gamma^{\prime}$ if $\gamma$ weakly Pareto dominates $\gamma^{\prime}$, and $\gamma\left(\mathcal{Z}, \succ_{C}, P_{S}\right)$ Pareto dominates $\gamma^{\prime}\left(\mathcal{Z}, \succ_{C}, P_{S}\right)$ for some $\left(\mathcal{Z}, \succ_{C}, P_{S}\right)$.

\section{Results}

\subsection{Common Priority among the Bottom Students}

First, we present the non-existence theorem of stable assignments. That is, there is a problem in which a feasible, fair and non-wasteful assignment does not exist for some preferences. Ehlers et al. (2014) show this result by proving the following two stronger statements: ${ }^{7}$

\footnotetext{
${ }^{7}$ Ehlers (2010) originally shows (ii) of Theorem 1 using the same example.
} 
Theorem 1. (Ehlers et al., 2014)

(i) The set of feasible assignments that are fair may be empty.

(ii) The set of feasible assignments that are both fair for same types and non-wasteful may be empty.

The proof is by means of an example. Example 1 is a counterexample for the first statement, and the second statement is shown by dropping $s_{3}$ from this problem.

Example 1. (Ehlers et al., 2014) Consider the following problem: $C=\left\{c_{1}, c_{2}, c_{3}\right\}, S=$ $\left\{s_{1}, s_{2}, s_{3}\right\}$ and $T=\left\{t_{1}, t_{2}\right\}$. Students $s_{1}$ and $s_{2}$ are of the same type and student $s_{3}$ 's type is different, i.e., $\tau\left(s_{1}\right)=\tau\left(s_{2}\right)=t_{1}$, and $\tau\left(s_{3}\right)=t_{2}$. Priorities, preferences, capacities and type-specific constraints are summarized in the table below. Note that $s_{3}$ 's preferences are arbitrary.

\begin{tabular}{ccccccc} 
& $\succ_{c_{1}}$ & $\succ_{c_{2}}$ & $\succ_{c_{3}}$ & $P_{s_{1}}$ & $P_{s_{2}}$ & $P_{s_{3}}$ \\
\hline & $s_{2}$ & $s_{2}$ & $s_{1}$ & $c_{2}$ & $c_{3}$ & $\vdots$ \\
& $s_{1}$ & $s_{1}$ & $s_{2}$ & $c_{3}$ & $c_{2}$ & \\
& $s_{3}$ & $s_{3}$ & $s_{3}$ & $c_{1}$ & $c_{1}$ & \\
capacity (ceiling for any type) & 1 & 1 & 1 & & & \\
floor for type $t_{1}$ & 1 & 0 & 0 & & & \\
floor for type $t_{2}$ & 0 & 0 & 0 & & & \\
\hline
\end{tabular}

As one of the type- $t_{1}$ students must be assigned to $c_{1}$, there are only four feasible assignments:

$$
\begin{aligned}
& \mu_{1}=\left(\begin{array}{lll}
c_{1} & c_{2} & c_{3} \\
s_{1} & s_{2} & s_{3}
\end{array}\right), \mu_{2}=\left(\begin{array}{lll}
c_{1} & c_{2} & c_{3} \\
s_{1} & s_{3} & s_{2}
\end{array}\right), \\
& \mu_{3}=\left(\begin{array}{lll}
c_{1} & c_{2} & c_{3} \\
s_{2} & s_{3} & s_{1}
\end{array}\right), \mu_{4}=\left(\begin{array}{lll}
c_{1} & c_{2} & c_{3} \\
s_{2} & s_{1} & s_{3}
\end{array}\right) .
\end{aligned}
$$

It is easy to see that none of them is fair:

1. $\mu_{1}$ is not fair as $s_{2}$ justifiably envies $s_{3}$ at $c_{3}$,

2. $\mu_{2}$ is not fair as $s_{1}$ justifiably envies $s_{2}$ at $c_{3}$,

3. $\mu_{3}$ is not fair as $s_{1}$ justifiably envies $s_{3}$ at $c_{2}$, and

4. $\mu_{4}$ is not fair as $s_{2}$ justifiably envies $s_{1}$ at $c_{2}$. 
Thus, there is no feasible and fair assignment.

Intuitively, only one of type- $t_{1}$ students can be assigned to $c_{2}$ or $c_{3}$ because of $c_{1}$ 's minimum quota. When a type- $t_{1}$ student is assigned to her second choice, she justifiably envies $s_{3}$ at her first-choice school. (When $s_{3}$ is not in the problem, this student justifiably claims an empty slot.) But when a type- $t_{1}$ student is assigned to her first choice, she is justifiably envied by the other type- $t_{1}$ student because the other type- $t_{1}$ student has a higher priority. In this way, there is a cycle of justified envy (and a claim for an empty slot for the second statement), and we cannot find a fair and non-wasteful assignment.

Given this impossibility theorem, several papers in the literature have proposed mechanisms that satisfy weaker requirements than fairness and non-wastefulness (Ehlers et al., 2014; Fragiadakis and Troyan, 2017; Goto et al., 2017). In their approach, they regard the constraints and priorities as given and try to find a solution for any given problem. But in many applications, the priority of schools could be designed as part of the designer's problem. On the other hand, students' preferences are not easily controlled by the designer. Therefore, in this paper, we seek a domain of schools' priority profiles that accommodate fair and non-wasteful assignments for any preferences of students given any primitive problem. ${ }^{8}$

The key feature of the priority structure in this example is that between $s_{1}$ and $s_{2}$, who are of the same type, $s_{1}$ has higher priority than $s_{2}$ at $c_{3}$ while $s_{2}$ has higher priority than $s_{1}$ at $c_{2}$. Combined with the minimum quota of $c_{1}$ and students' preferences, this causes a cycle of justified envy. Since type-specific constraints are given as part of the primitive problem and we consider the whole domain of students' preferences, we need to design schools' priority so that $c_{2}$ and $c_{3}$ both give higher priority to the same student, either $s_{1}$ or $s_{2}$.

Motivated by this observation, we first propose a strong property of schools' priority rankings: common priority.

Definition 4. A pair of priority rankings $\left(\succ_{c}, \succ_{c^{\prime}}\right)$ of two schools $c$ and $c^{\prime}$ has a common priority order for type $t \in T$ if

$$
s \succ_{c} s^{\prime} \Leftrightarrow s \succ_{c^{\prime}} s^{\prime}
$$

for any $s, s^{\prime} \in S_{t}$.

The common priority condition says that the priority rankings among the same type students are exactly the same between given two schools. Notice that the ranking between

\footnotetext{
${ }^{8}$ It is also possible to consider the domain of type-specific constraints that ensures the existence of fair and non-wasteful assignments for any preferences of students given the schools' priority structure. Since our theorems disclose the relationship between priority and constraints, this problem might be solved by exploiting our results.
} 
different types of students can differ between these schools in an arbitrary way. It is easy to see that in Example 1, we would find a fair and non-wasteful assignment if $c_{2}$ and $c_{3}$ had a common priority order for type $t_{1}$.

Although common priority is easy to understand, this condition may not be necessary for the existence of stable assignments depending on the number of minimum quotas. In particular, when the minimum quota is low enough, the ranking among some top students does not matter. We can see this in the following example.

Example 2. Consider the following problem: $C=\left\{c_{1}, c_{2}, c_{3}\right\}, S=\left\{s_{1}, s_{2}, s_{3}\right\}$ and $T=\left\{t_{1}\right\}$. All students are of the same type. Priorities, capacities and type-specific constraints are summarized in the table below.

\begin{tabular}{cccc} 
& $\succ_{c_{1}}$ & $\succ_{c_{2}}$ & $\succ_{c_{3}}$ \\
\hline & $s_{2}$ & $s_{2}$ & $s_{1}$ \\
& $s_{1}$ & $s_{1}$ & $s_{2}$ \\
& $s_{3}$ & $s_{3}$ & $s_{3}$ \\
capacity (ceiling for type $\left.t_{1}\right)$ & 1 & 2 & 2 \\
floor for type $t_{1}$ & 1 & 0 & 0 \\
\hline
\end{tabular}

We can see that $c_{3}$ and other schools do not have a common priority order for type $t_{1}$. However, we can always find a fair and non-wasteful assignment for these capacity constraints. First, $s_{1}$ and $s_{2}$ can be assigned to schools so that none of them justifiably envies the other or claims an empty slot of any other school. Next, $s_{3}$ is assigned to $c_{1}$ if $c_{1}$ 's minimum quota is not filled by $s_{1}$ or $s_{2}$, and otherwise, $s_{3}$ can choose the best available slot.

In this example, a stable assignment exists for any preferences because $s_{3}$ fills $c_{1}$ 's minimum quota if it is not filled by other students. The key feature is that there is only one slot in the minimum quota of $c_{1}$, and all schools agree on the bottom one student $s_{3}$. This gives the idea of $B$-common priority, which requires the consistency of priority rankings "only among certain bottom students."

For the definition of B-common priority, let us define $F(\tilde{C}, t)$ for any $\tilde{C} \subseteq C$ with $|\tilde{C}| \leq$ $m-2$ and $t \in T . F(\tilde{C}, t)$ is the set of feasible assignments under which the type- $t$ minimum quotas of all schools in $\tilde{C} \subseteq C$ are binding. More formally,

$$
F(\tilde{C}, t) \equiv\left\{\mu \in F|| \mu^{t}(c) \mid=\underline{q}_{c}^{t} \text { for all } c \in \tilde{C}\right\} .
$$

Also, for each $\succ_{c}$ and $K \in\left\{0,1,2, \ldots,\left|S_{t}\right|\right\}$, let $B_{t}\left(\succ_{c}, K\right) \equiv\left\{s \in S_{t}||\left\{s^{\prime} \in S_{t} \mid s \succ_{c} s^{\prime}\right\} \mid<K\right\}$ be the bottom $K$ students of type $t$ according to $\succ_{c}$. In the same way, for each strict ranking 
$r^{t}: S_{t} \rightarrow\left\{1,2, \ldots,\left|S_{t}\right|\right\}$ over type- $t$ students and $K \in\left\{0,1,2, \ldots,\left|S_{t}\right|\right\}$, let $B_{t}\left(r^{t}, K\right) \equiv\{s \in$ $\left.S_{t} \|\left\{s^{\prime} \in S_{t} \mid r^{t}(s)<r^{t}\left(s^{\prime}\right)\right\} \mid<K\right\}$ be the bottom $K$ students of type $t$ in the ranking $r^{t}{ }^{9}$ Note that $B_{t}\left(\succ_{c}, 0\right)=B_{t}\left(r^{t}, 0\right)=\emptyset$ for any $t \in T, \succ_{c}$ and $r^{t}$.

Definition 5. A priority profile of schools $\succ_{C}$ has common priority among the bottom students (B-common priority) if for each type $t \in T$, there exists a strict ranking $r^{t}$ : $S_{t} \rightarrow\left\{1,2, \ldots,\left|S_{t}\right|\right\}$ over type- $t$ students such that for any school $c \in C$,

$$
B_{t}\left(\succ_{c}, K\right)=B_{t}\left(r^{t}, K\right)
$$

holds for any $K \in\left\{0, \ldots, Q_{t}(c)\right\}$, where

$$
\begin{aligned}
& Q_{t}(c) \equiv \max _{c^{\prime} \in C \backslash\{c\}} Q_{t}\left(c, c^{\prime}\right), \quad \text { and } \\
& Q_{t}\left(c, c^{\prime}\right) \equiv \max _{\tilde{C} \in\left\{C^{\prime} \subseteq C \backslash\left\{c c^{\prime}\right\} \mid F\left(C^{\prime}, t\right) \neq \emptyset\right\}} \sum_{c^{\prime \prime} \in \tilde{C}} q_{c^{\prime \prime}}^{t} .
\end{aligned}
$$

Under the B-common priority condition, each school c's priority ranking is consistent with a single ranking $r^{t}$ for all the bottom $Q_{t}(c)$ students. The threshold $Q_{t}(c)$ varies across schools, and it is computed by the capacities and type-specific constraints of all schools. We define $Q_{t}(c)$ by taking the maximum of $Q_{t}\left(c, c^{\prime}\right)$ over all other schools in $C \backslash\{c\}$. This means that for each pair of schools $\left(c, c^{\prime}\right)$, priority rankings of the bottom $Q_{t}\left(c, c^{\prime}\right)$ type- $t$ students are exactly the same between two schools $c$ and $c^{\prime}$. Notice that the top $\left|S_{t}\right|-Q_{t}\left(c, c^{\prime}\right)$ type- $t$ students may be ranked in a different way between $c$ and $c^{\prime}$. Therefore, for each pair of schools, this requirement is a weaker than common priority in Definition 4.

Then, what does $Q_{t}\left(c, c^{\prime}\right)$ mean? $Q_{t}\left(c, c^{\prime}\right)$ is the maximum of the sum of all binding type- $t$ minimum quotas in $C \backslash\left\{c, c^{\prime}\right\}$ among feasible assignments. In Example $2, Q_{t_{1}}\left(c_{2}, c_{3}\right)$ is computed as one. Although $\left(\succ_{c_{2}}, \succ_{c_{3}}\right)$ does not have a common priority order for type $t_{1}, c_{2}$ and $c_{3}$ agree on the bottom one student, who is $s_{3}$ in this case. Since $Q_{t_{1}}\left(c_{1}, c_{2}\right)=$ $Q_{t_{1}}\left(c_{1}, c_{3}\right)=0$, there is no restriction on $\succ_{c_{1}}$ and we say that the problem in Example 2 has B-common priority. Intuitively, common priority among the bottom $Q_{t}\left(c, c^{\prime}\right)$ students is needed in the worst-case scenario where all type- $t$ students prefer $c$ or $c^{\prime}$ and we need to fill $Q_{t}\left(c, c^{\prime}\right)$ minimum quotas in $C \backslash\left\{c, c^{\prime}\right\}$.

$Q_{t}\left(c, c^{\prime}\right)$ can be easily computed as $Q_{t}\left(c, c^{\prime}\right)=\sum_{c^{\prime \prime} \in C \backslash\left\{c, c^{\prime}\right\}} \underline{q}_{c^{\prime \prime}}^{t}$ when there is a feasible assignment in which all type- $t$ minimum quotas are binding. As we see in Section 4 , this is often the case in applications. For other cases, we also give an algorithm to compute $Q_{t}\left(c, c^{\prime}\right)$ for each pair of schools $\left(c, c^{\prime}\right)$ and each type $t$ in Appendix C.

\footnotetext{
${ }^{9}$ The interpretation of $r^{t}(s)$ is the "ranking" of student $s$. If student $s$ is ranked higher in $r^{t}$, then $s$ has a smaller number $r^{t}(s)$. We also write $r^{t}: s, s^{\prime}, \cdots$ to mean that $r^{t}(s)=1, r^{t}\left(s^{\prime}\right)=2$ and so on.
} 
The B-common priority condition is considered as a variant of the acyclicity conditions (Ergin, 2002; Kesten, 2006; Haeringer and Klijn, 2009; Kumano, 2013). Our condition, however, is not equivalent to them. For example, Ergin (2002) showed that his acyclicity condition is equivalent to requiring that the ranks of certain bottom students differ at most by one between two schools (Theorem 2, Ergin, 2002). On the other hand, the B-common priority condition requires that if a student is ranked below a certain threshold, she must have the same rank across schools among the same type students. In addition, the threshold is computed by the capacity and type-specific constraints of all schools in our definition.

Before showing the main results, let us discuss the following question: How likely is the (B-)common priority condition satisfied in real-world applications? In college admissions and student-supervisor matching problems, it is often the case that there is a natural ranking of students that all schools can use. For example, in several countries such as Australia and China, all students are ranked by a single score in the centralized college admission system. When assigning students to supervisors in universities, GPA is often used as a natural priority ranking. Moreover, B-common priority would give schools a freedom to rank some top students as they like; depending on the number of minimum quotas, they can reflect their heterogeneous preferences over good students to their rankings.

On the other hand, in (elementary, middle and high) school choice programs, common priority for same type students may be unlikely to be met for a typical choice of types and priority structures. Consider school districts which require gender-specific constraints and introduce walk-zone priority. Walk-zone priority is used in Boston Public Schools, New York City High School Match and many other programs. In such cases, it is hard to guarantee common priority for same type students even among the bottom students because two female (male) students can live in different walk-zones, and hence the priority order of these students may be reversed between different schools.

These observations suggest that our existence theorem may or may not be applied depending on the background of the problem. As we will see, however, our results have an implication to problems without B-common priority as well. First, as Theorem 3 shows the necessity of the pairwise version of B-common priority (in a maximal domain sense), we know that we have to give up stability for some preferences of students in such cases. Next, since our DAPL has good properties for all problems (Propositions 1, 3 and 4), DAPL may improve the current mechanism for the relevant application. 


\subsection{DA with Precedence Lists}

In this subsection, we show that we can always find a fair and non-wasteful assignment for problems with B-common priority. To do so, we propose a new mechanism called Deferred Acceptance Mechanism with Precedence Lists (DAPL).

To define DAPL, we specify "precedence lists" for each type in each problem. ${ }^{10}$ A precedence list for type $t$ is a strict ranking $r^{t}: S_{t} \rightarrow\left\{1,2, \ldots,\left|S_{t}\right|\right\}$ over type- $t$ students. For any problem with B-common priority, we set each $r^{t}$ to satisfy

$$
B_{t}\left(\succ_{c}, K\right)=B_{t}\left(r^{t}, K\right)
$$

for any $c \in C$ and any $K \in\left\{0, \ldots, Q_{t}(c)\right\}$. For any other problem, the precedence lists are defined arbitrarily.

The basic idea of DAPL is to transform a problem into a corresponding problem with only maximum quotas. We consider a null division for each type which students must propose to in Step 1. The priority order of the type- $t$ null division is defined by the reverse order of the precedence list $r^{t}$. If they are rejected by null divisions, they can propose to (the divisions of) schools. If they remain in the null division after the DA stage, they must fill the minimum quotas that are not filled yet. To implement this idea, we define the divisions of schools, and also introduce artificial students to identify who are allowed to propose to schools and who must fill the minimum quotas.

Let $Q_{t}$ be the maximum of $Q_{t}(c)$ over all schools, i.e., $Q_{t} \equiv \max _{c \in C} Q_{t}(c)$.

\section{Deferred Acceptance Mechanism with Precedence Lists}

Start: For each type $t \in T$, add $Q_{t}$ (identical) artificial students. The set of such students is denoted by $A_{t}$. Let $A \equiv \cup_{t \in T} A_{t}$. Instead of schools, we consider the following three types of divisions: the type- $t$ division $c^{t}$ of school $c$ for each $(t, c) \in T \times C$; the open division $c^{o}$ of school $c$ for each $c \in C$; and the type- $t$ null division $\phi^{t}$ for each $t \in T$. Each of these divisions has the following number of slots and an extended priority order over real and artificial students in $S \cup A$.

- The type- $t$ division $c^{t}$ of school $c$ has $\underline{q}_{c}^{t}$ slots. The extended priority order $\succ_{c^{t}}$ must satisfy (i) $s \succ_{c^{t}} a \succ_{c^{t}} x$ for any $s \in S_{t}, a \in A_{t}$ and $x \in(S \cup A) \backslash\left(S_{t} \cup A_{t}\right)$, and (ii) $s \succ_{c^{t}} s^{\prime}$ for any $s, s^{\prime} \in S_{t}$ such that $s \succ_{c} s^{\prime}$.

\footnotetext{
${ }^{10}$ We borrowed the name "precedence list" from the multi-stage deferred acceptance mechanism (MSDA) proposed by Fragiadakis et al. (2015). See subsection 3.4 for the comparison between MSDA and DAPL. In Appendix D, we also examine the properties of the serial dictatorship mechanism in this problem.
} 
- The open division $c^{o}$ of school $c$ has $q_{c}-\sum_{t \in T} \underline{q}_{c}^{t}$ slots. The extended priority order $\succ_{c^{o}}$ must satisfy $s \succ_{c^{o}} s^{\prime}$ for any $s, s^{\prime} \in S$ such that $s \succ_{c} s^{\prime}$.

- The type- $t$ null division $\phi^{t}$ has $Q_{t}$ slots. The extended priority order $\succ_{\phi^{t}}$ must satisfy (i) $a \succ_{\phi^{t}} s \succ_{\phi^{t}} x$ for any $a \in A_{t}, s \in S_{t}$ and $x \in(S \cup A) \backslash\left(S_{t} \cup A_{t}\right)$, and (ii) $s^{\prime} \succ_{\phi^{t}} s$ for any $s, s^{\prime} \in S_{t}$ such that $r^{t}(s)<r^{t}\left(s^{\prime}\right)$.

We also define the extended preferences $\tilde{P}_{S \cup A}$ of all students in $S \cup A$ over these divisions.

- For any real student $s \in S_{t}$, transform her submitted preference $P_{s}$ over schools into an extended preference $\tilde{P}_{s}$ over divisions in the following way: (i) $\phi^{t} \tilde{P}_{s} c^{t} \tilde{P}_{s} c^{o} \tilde{P}_{s} \phi^{\tilde{t}} \tilde{P}_{s} c^{\tilde{t}}$ for any $c \in C$ and $\tilde{t} \neq t$, and (ii) $c^{i} \tilde{P}_{s} \tilde{c}^{j}$ for any $i, j \in\{t, o\}$ and $c, \tilde{c} \in C$ such that $c P_{s} \tilde{c}$.

- For any artificial student $a \in A_{t}$, assume that $c_{1}^{t} \tilde{P}_{a} \ldots \tilde{P}_{a} c_{m}^{t} \tilde{P}_{a} \phi^{t} \tilde{P}_{a} d$ for any other division $d$.

DA stage: Run the following version of the student-proposing Deferred Acceptance mechanism with type-specific maximum quotas between all (real and artificial) students and divisions using extended preferences and priority orders.

Step p: Each student who is not tentatively accepted by any division proposes to her next most preferred division. Each division considers students who are tentatively accepted to it and the new applicants in this step.

- Each $c^{t}$ tentatively accepts students among them up to $\underline{q}_{c}^{t}$ according to its extended priority order $\succ_{c^{t}}$.

- Each $c^{o}$ examines them one by one according to $\succ_{c^{o}}$. If a student is of type $t$, it tentatively accepts her if $q_{c}-\sum_{t \in T} \underline{q}_{c}^{t}$ seats are not filled and $\min \left\{\bar{q}_{c}^{t}-\right.$ $\left.\underline{q}_{c}^{t},\left|S_{t}\right|-\sum_{c^{\prime} \in C} \underline{q}_{c^{\prime}}^{t}\right\}$ seats are not filled by type- $t$ students.

- Each $\phi^{t}$ tentatively accepts students among them up to $Q_{t}$ according to its extended priority order $\succ_{\phi^{t}}$.

Each division rejects all other applicants.

If some student is rejected in step $p$, proceed to step $p+1$. When no student is rejected, proceed to the additional rejection step. ${ }^{11}$

\footnotetext{
${ }^{11}$ We need these steps to show the fairness of DAPL in Proposition 2. More details are discussed following Proposition 2.
} 
Additional Rejection Step: Out of all type- $t$ divisions $\left\{c^{t}\right\}_{c \in C}$, if only one $c_{l}^{t}$ is fully filled by real students, then reject one type- $t$ artificial student $a \in A_{t}$ from $c_{m}^{t}$ if $l \neq m$ or from $c_{m-1}^{t}$ if $l=m .{ }^{12}$ Do the same procedure for each type $t$. Then, go back to a normal step of the DA stage again.

The DA stage terminates when no student is rejected and there is no additional rejection step. The slots of all school divisions for real students are finalized.

Minimum Quota stage: For each type $t \in T$, if there are some slots of type- $t$ divisions currently held by artificial students, we assign these slots to the real students who have slots of the null division $\phi^{t}$ using the serial dictatorship. That is, each of these students picks her most preferred school sequentially according to the precedence list $r^{t}$ (a student with smaller number $r^{t}(\cdot)$ picks a school earlier.) And she is assigned a slot of the school if an artificial student currently holds it in its type- $t$ division. ${ }^{13}$

We illustrate how DAPL works using a simple example of a problem without B-common priority.

Example 3. Consider the following problem: $C=\left\{c_{1}, c_{2}, c_{3}\right\}, S=\left\{s_{1}, s_{2}, s_{3}\right\}$ and $T=$ $\left\{t_{1}\right\}$. All students are of the same type. Priorities, preferences, capacities and type-specific constraints are summarized in the table below.

\begin{tabular}{ccccccc} 
& $\succ_{c_{1}}$ & $\succ_{c_{2}}$ & $\succ_{c_{3}}$ & $P_{s_{1}}$ & $P_{s_{2}}$ & $P_{s_{3}}$ \\
\hline & $s_{1}$ & $s_{1}$ & $s_{1}$ & $c_{1}$ & $c_{2}$ & $c_{3}$ \\
& $s_{2}$ & $s_{3}$ & $s_{2}$ & $\vdots$ & $c_{3}$ & $c_{2}$ \\
& $s_{3}$ & $s_{2}$ & $s_{3}$ & & $c_{1}$ & $c_{1}$ \\
capacity (ceiling for type $\left.t_{1}\right)$ & 3 & 1 & 1 & & & \\
floor for type $t_{1}$ & 2 & 0 & 0 & & & \\
\hline
\end{tabular}

Note that $\succ_{C}$ does not have B-common priority because we have $Q_{t_{1}}\left(c_{2}, c_{3}\right)=2$ and the bottom student is different between $\succ_{c_{2}}$ and $\succ_{c_{3}}$. Consider the following precedence list $r^{t_{1}}: s_{1}, s_{2}, s_{3}$. At the beginning of DAPL, we create the divisions. As the only minimum quota is imposed on $c_{1}$, we only need to consider two divisions for $c_{1}\left(c_{1}^{t_{1}}\right.$ and $\left.c_{1}^{o}\right)$, one

\footnotetext{
${ }^{12}$ If a type division has zero quota, then we interpret this as being filled by real students. Also by the definition of the capacities of open divisions, for any type $t$, there is always one school whose type- $t$ division is fully filled by real students.

${ }^{13}$ This stage is well-defined because the number of real students in the null division $\phi^{t}$ and the total number of slots of type- $t$ divisions occupied by artificial students must be the same. In addition, we show in Proposition 1 that DAPL is always feasible, i.e., minimum quotas are always filled.
} 
division for each of the other two schools $\left(c_{2}^{o}\right.$ and $\left.c_{3}^{o}\right)$, and the null division for $t_{1}\left(\phi^{t_{1}}\right)$. By $Q_{t_{1}}=Q_{t_{1}}\left(c_{2}, c_{3}\right)=2$, two artificial students $a_{1}, a_{2} \in A_{t_{1}}$ are added to the market. The extended priority orders and preferences are as follows:

\begin{tabular}{ccccccccccc}
$\succ_{c_{1}^{t_{1}}}$ & $\succ_{c_{1}^{o}}$ & $\succ_{c_{2}^{o}}$ & $\succ_{c_{3}^{o}}$ & $\succ_{\phi^{t_{1}}}$ & $\tilde{P}_{s_{1}}$ & $\tilde{P}_{s_{2}}$ & $\tilde{P}_{s_{3}}$ & $\tilde{P}_{a_{1}}$ & $\tilde{P}_{a_{2}}$ \\
\hline$s_{1}$ & $s_{1}$ & $s_{1}$ & $s_{1}$ & $a_{1}$ & $\phi^{t_{1}}$ & $\phi^{t_{1}}$ & $\phi^{t_{1}}$ & $c_{1}^{t_{1}}$ & $c_{1}^{t_{1}}$ \\
$s_{2}$ & $s_{2}$ & $s_{3}$ & $s_{2}$ & $a_{2}$ & $c_{1}^{t_{1}}$ & $c_{2}^{o}$ & $c_{3}^{o}$ & $\phi^{t_{1}}$ & $\phi^{t_{1}}$ \\
$s_{3}$ & $s_{3}$ & $s_{2}$ & $s_{3}$ & $s_{3}$ & $c_{1}^{o}$ & $c_{3}^{o}$ & $c_{2}^{o}$ & $\vdots$ & $\vdots$ \\
$a_{1}$ & $a_{1}$ & $a_{1}$ & $a_{1}$ & $s_{2}$ & $\vdots$ & $c_{1}^{t_{1}}$ & $c_{1}^{t_{1}}$ & & & \\
$a_{2}$ & $a_{2}$ & $a_{2}$ & $a_{2}$ & $s_{1}$ & & $c_{1}^{o}$ & $c_{1}^{o}$ & &
\end{tabular}

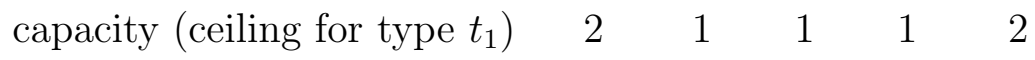

In the DA stage, we run the student-proposing DA mechanism between students $\left\{s_{1}, s_{2}, s_{3}, a_{1}, a_{2}\right\}$ and divisions $\left\{c_{1}^{t_{1}}, c_{1}^{o}, c_{2}^{o}, c_{3}^{o}, \phi^{t_{1}}\right\}$.

- In Step 1, all real students propose to $\phi^{t_{1}}$, and all artificial students propose to $c_{1}^{t_{1}} \cdot \phi^{t_{1}}$ temporarily accepts $s_{2}$ and $s_{3}$, and rejects $s_{1}$. $c_{1}^{t_{1}}$ temporarily accepts $a_{1}$ and $a_{2}$.

- In Step 2, $s_{1}$ proposes to $c_{1}^{t_{1}}$. $c_{1}^{t_{1}}$ temporarily accepts $s_{1}$ and $a_{1}$, and rejects $a_{2}$.

- In Step 3, $a_{2}$ proposes to $\phi^{t_{1}}$. $\phi^{t_{1}}$ temporarily accepts $a_{2}$ and $s_{3}$, and rejects $s_{2}$.

- In Step $4, s_{2}$ proposes to $c_{2}^{o}$ and is temporarily accepted.

Now as every student is assigned and more than one schools' minimum quotas are filled by real students $\left(c_{2}\right.$ and $c_{3}$ ), the DA stage is done. In the Minimum Quota stage, since $s_{3}$ is the only student who was assigned to the null division, $s_{3}$ is assigned $a_{1}$ 's slot at $c_{1}^{t_{1}}$. The final assignment is

$$
\mu^{*}=\left(\begin{array}{ccc}
c_{1} & c_{2} & c_{3} \\
\left\{s_{1}, s_{3}\right\} & s_{2} & \emptyset
\end{array}\right)
$$

which is feasible. It is easy to see that this assignment is non-wasteful because $s_{1}$ and $s_{2}$ are assigned to their top choices and $s_{3}$ cannot justifiably claim an empty slot at $c_{3}$. $\mu^{*}$ is not fair for same types because $s_{3}$ justifiably envies $s_{2}$ at $c_{3}$. But $s_{3}$ is the only student who has justified envy.

The key of this algorithm is as follows. In Example 3, by making all type- $t_{1}$ students propose to $\phi^{t_{1}}$ first, we can ensure that there are enough students who finally fill the minimum quota of school $c_{1}$. However, when a type- $t_{1}$ student (in this case, student $s_{1}$ ) fills a slot of 
$c_{1}$, which has a minimum quota for $t_{1}$, we have to release another type- $t_{1}$ student (in this case, $\left.s_{2}\right)$ from $\phi^{t_{1}}$ to ensure non-wastefulness. To do so, we let every student propose to the type division of each school before proposing to its open division. In other words, if $s_{1}$ were assigned a slot of $c_{1}^{o}$, then both $s_{2}$ and $s_{3}$ would be assigned to $c_{1}^{t_{1}}$ in the MQ stage, and this would generate justified claims of empty slots by $s_{2}$ and $s_{3}$. The artificial students help us count the number of minimum quotas we need to fill and identify who need to fill them. Once artificial students are rejected by the divisions of schools and go to null divisions, they release real students from null divisions and allow them to propose to the school divisions. In addition, the priority order of each null division is defined to be the reverse order of the precedence list $r^{t}$ for each $t$. Because of this, higher a student is ranked under $r^{t}$, earlier she is rejected by the null division and proposes to other divisions.

$\mu^{*}$ is not fair because $s_{3}$ justifiably envies $s_{2}$ who is of the same type. ${ }^{14}$ This happens because although $s_{3}$ has higher priority than $s_{2}$ at $c_{2}, s_{3}$ is ranked lowest in the precedence list $r^{t}$ and could not propose to any school divisions in the DA stage. This observation can be generalized: in the outcome of DAPL, if some student justifiably envies another, then this student must have been assigned to the null division in the DA stage.

Now we formalize this idea to introduce a weaker version of fairness that is satisfied by DAPL. We call this new fairness strict PL-fairness. For problems with only one type, Fragiadakis et al. (2015) introduced PL-fairness, which is weaker than our strict PL-fairness. ${ }^{15}$

Definition 6. Student s PL-envies student s' of the same type at school c under an assignment $\mu$ if

$$
\begin{aligned}
& \left(\mathrm{f} 1^{* *}\right) \mu\left(s^{\prime}\right)=c, c P_{s} \mu(s), \text { and } s \succ_{c} s^{\prime}, \\
& \left(\mathrm{f} 2^{* *}\right) \tau(s)=\tau\left(s^{\prime}\right), \text { and } \\
& \left(\mathrm{f} 3^{* *}\right) r^{\tau(s)}(s)<r^{\tau(s)}\left(s^{\prime}\right), s \notin B_{\tau(s)}\left(r^{\tau(s)}, Q_{\tau(s)}\right), \text { or }\left|\mu_{\tau(s)}(\mu(s))\right|>\underline{q}_{\mu(s)}^{\tau(s)} .
\end{aligned}
$$

A feasible assignment $\mu$ is strictly PL-fair for same types if there is no student who PL-envies any other student of the same type.

$\left(\mathrm{f} 1^{* *}\right)$ and $\left(\mathrm{f} 2^{* *}\right)$ are the same as standard envy for same type students. ( $\left.\mathrm{f} 3^{* *}\right)$ is the additional requirement for PL-envy. ( $\left.f 3^{* *}\right)$ implies that in order for student $s$ to PL-envy student $s^{\prime}$ of the same type, at least one of the three following conditions must be satisfied:

\footnotetext{
${ }^{14}$ Indeed, this example has a similar structure as Example 1 and does not have any feasible assignment that is fair and non-wasteful.

${ }^{15}$ For PL-fairness, $\left(\mathrm{f} 3^{* *}\right)$ is strengthened to only $r^{\tau(s)}(s)<r^{\tau(s)}\left(s^{\prime}\right)$.
} 
(i) $s$ is ranked higher than $s^{\prime}$ according to $r^{\tau(s)}$; (ii) $s$ is not one of the bottom $Q_{\tau(s)}$ students in the precedence list $r^{\tau(s)}$; or (iii) the number of type- $t$ students assigned to $\mu(s)$ under $\mu$ is strictly greater than the minimum quota $\underline{q}_{\mu(s)}^{\tau(s)}$. We say that a feasible assignment $\mu$ is strictly $P L$-fair if it is both fair across different types and strictly PL-fair for same types.

Strict PL-fairness is also described in the following way: The only justified envy that cannot be eliminated by strict PL-fairness is such that (i) $s$ justifiably envies $s^{\prime}$ who is of the same type and ranked higher than $s$ according to $r^{\tau(s)}$; (ii) $s$ is one of the bottom $Q_{\tau(s)}$ students in the precedence list $r^{\tau(s)}$; and (iii) the minimum quota of $\mu(s)$ for type- $t$ students is binding at $\mu$.

The next proposition summarizes the properties of DAPL which hold for any problem.

Proposition 1. DAPL is

\section{1. feasible;}

2. non-wasteful;

3. fair across different types;

4. strictly PL-fair for same types; and

\section{5. group strategy-proof.}

For the feasibility of DAPL, we show that $Q_{t}$ artificial students are enough to fill all minimum quotas of type- $t$ divisions in the DA stage for any preferences. For non-wastefulness and strict PL-fairness, first we can see that a student does not justifiably claim an empty slot or envy another student if she is assigned a school's slot in the DA stage. In addition, even when a student is assigned a slot of a null division in the DA stage, she cannot justifiably claim an empty slot. Thus, we only need to characterize possible envy of students who are assigned to null divisions, and show the strict PL-fairness of DAPL.

For group strategy-proofness, we start from the observation that DAPL is constructed from DA with type-specific maximum quotas (Abdulkadiroğlu, 2005) by (i) applying it to the DA stage and introducing additional rejection steps, and (ii) adding the MQ stage after the DA stage. Since DA with type-specific maximum quotas is group strategy-proof (Hatfield and Kojima, 2009), we show that these two extensions do not harm the incentives of students. Theorem 3 of Ehlers et al. (2014) states that there is no feasible mechanism that is strategyproof, fair for same types and constrained non-wasteful. Contrary to this negative result, we show that strategy-proofness can be restored by weakening fairness for same types to strict 
PL-fairness for same types. In addition, DAPL satisfies non-wastefulness and fairness across different types, which are weakened or dropped in Theorem 3 of Ehlers et al. (2014).

The next result provides a sufficient condition for DAPL to be fair as well.

Proposition 2. For problems with B-common priority, DAPL is fair.

Since DAPL is always strictly PL-fair, this proposition shows that under B-common priority, no student justifiably envies another student of the same type even if she is assigned a slot in the MQ stage. We consider two cases such that (i) $c$ is fully filled by real students in the DA stage and (ii) $c$ is not fully filled by real students in the DA stage. In each case, B-common priority ensures that no student justifiably envies another student of same type who is admitted to $c$.

For the proof of Proposition 2, additional rejection steps are essential. ${ }^{16}$ If we removed additional rejection steps from DAPL, it would fail to achieve fairness in problems with B-common priority. Example 6 in Appendix D illustrates this point.

Our first existence theorem is immediately obtained from Propositions 1 and 2.

Theorem 2. For any problem with B-common priority, there exists a feasible, fair and non-wasteful assignment.

\subsection{Efficiency of DAPL}

In this subsection, we examine the efficiency of DAPL. In special cases where constraints are not type-specific, i.e., $|T|=1$, we can show that there is no stable assignment that Pareto dominates the outcome of DAPL.

Proposition 3. For any problem with $|T|=1$, the outcome of DAPL is not Pareto dominated by any feasible, fair and non-wasteful assignment.

First, we show that if the outcome of DAPL is Pareto dominated by a fair and nonwasteful assignment, there must be a fair and non-wasteful assignment that strictly improves the outcome of DAPL only for those who are assigned in the DA stage. But then by $|T|=1$, there is a corresponding stable assignment in the extended problem in the DA stage, and this leads to a contradiction to the "student optimality" of the standard DA mechanism.

But Proposition 3 does not continue to hold for $|T| \geq 2$ since some justified envy in the extended problem is not justified in the original problem when there are multiple types.

\footnotetext{
${ }^{16}$ Additional rejection steps are needed to show Lemma 1 in Appendix A, which is used for the proof of this proposition.
} 
Observation 1. There is a problem for which the outcome of DAPL is Pareto dominated by a feasible, fair and non-wasteful assignment.

Example 4. Consider the following problem: $C=\left\{c_{1}, c_{2}, c_{3}, c_{4}\right\}, S=\left\{s_{1}, s_{2}, s_{3}, s_{4}\right\}, T=$ $\left\{t_{1}, t_{2}\right\}, \tau\left(s_{1}\right)=\tau\left(s_{2}\right)=t_{1}$, and $\tau\left(s_{3}\right)=\tau\left(s_{4}\right)=t_{2}$. Priorities, preferences, capacities and type-specific constraints are summarized in the table below.

\begin{tabular}{ccccccccc} 
& $\succ_{c_{1}}$ & $\succ_{c_{2}}$ & $\succ_{c_{3}}$ & $\succ_{c_{4}}$ & $P_{s_{1}}$ & $P_{s_{2}}$ & $P_{s_{3}}$ & $P_{s_{4}}$ \\
\hline & $s_{2}$ & $s_{1}$ & $s_{3}$ & $s_{3}$ & $c_{1}$ & $c_{2}$ & $c_{1}$ & $c_{4}$ \\
& $s_{3}$ & $s_{3}$ & $s_{4}$ & $s_{4}$ & $c_{2}$ & $c_{1}$ & $c_{2}$ & $c_{3}$ \\
& $s_{4}$ & $s_{4}$ & $\vdots$ & $\vdots$ & $c_{4}$ & $c_{4}$ & $c_{3}$ & $\vdots$ \\
& $s_{1}$ & $s_{2}$ & & & $c_{3}$ & $c_{3}$ & $c_{4}$ & \\
capacity (ceiling for any type) & 1 & 1 & 1 & 1 & & & & \\
floor for type $t_{1}$ & 0 & 0 & 0 & 0 & & & & \\
floor for type $t_{2}$ & 0 & 0 & 1 & 0 & & & & \\
\hline
\end{tabular}

Note that $\succ_{C}$ has B-common priority in this problem. In DAPL, the precedence list for type $t_{2}$ must be $r^{t_{2}}: s_{3}, s_{4}$ because $Q_{t_{2}}=1$.

DAPL achieves the following assignment:

$$
\mu^{D A P L}=\left(\begin{array}{cccc}
c_{1} & c_{2} & c_{3} & c_{4} \\
s_{2} & s_{1} & s_{3} & s_{4}
\end{array}\right)
$$

However, this is Pareto dominated by the following assignment:

$$
\mu=\left(\begin{array}{cccc}
c_{1} & c_{2} & c_{3} & c_{4} \\
s_{1} & s_{2} & s_{3} & s_{4}
\end{array}\right)
$$

It is clear that $\mu$ is non-wasteful. Since $s_{3}$ cannot justifiably envy $s_{1}$ or $s_{2}$ because of the floor of $c_{3}$ for type $t_{2}, \mu$ is fair.

Since $s_{3}$ is not the bottom $Q_{t_{2}}=1$ student in $r^{t_{2}}$, she proposes to $c_{1}^{o}$ in the DA stage. But this generates a rejection chain and both $s_{1}$ and $s_{2}$ are assigned to their second choices. $s_{3}$ is finally assigned to $c_{3}$, but since $s_{1}$ and $s_{2}$ are of different types and $s_{3}$ just fills the minimum quota of $c_{3}$ for type $t_{2}, s_{3}$ 's envy to $s_{1}$ or $s_{2}$ is not justified according to Definition 2. The logic is similar to the idea that the student-optimal stable assignment may not be Pareto efficient for problems with no minimum quotas, but a stark difference is that $\mu$ is also fair in our problem.

To see if DAPL can be Pareto improved without harming fairness or non-wastefulness, we consider the following concept: Pareto dominance whenever stable improvement is possible. 
Definition 7. A mechanism $\gamma$ Pareto dominates another mechanism $\varphi$ whenever stable improvement is possible if

1. $\gamma$ weakly Pareto dominates $\varphi$; and

2. $\gamma\left(\mathcal{Z}, \succ_{C}, P_{S}\right)$ Pareto dominates $\varphi\left(\mathcal{Z}, \succ_{C}, P_{S}\right)$ for any $\left(\mathcal{Z}, \succ_{C}, P_{S}\right)$ such that $\varphi\left(\mathcal{Z}, \succ_{C}\right.$ ,$\left.P_{S}\right)$ is Pareto dominated by another feasible, fair and non-wasteful assignment.

Besides $\gamma$ weakly Pareto dominating $\varphi$, the only requirement of this concept is that the outcome of $\gamma$ Pareto dominates that of $\varphi$ whenever $\varphi$ exhibits inefficiency concerned in Observation 1. And this does not require that $\gamma$ should be Pareto efficient.

Our next result shows that it is impossible to improve DAPL according to this concept unless we give up strategy-proofness.

Proposition 4. There exists no strategy-proof mechanism that Pareto dominates DAPL whenever stable improvement is possible.

Proof uses Example 4 to show that no mechanism can be strategy-proof if it selects $\mu$ and weakly Pareto dominates DAPL. The idea is similar to Proposition 1 of Kesten (2010), but we make a careful adjustment to our definitions of fairness. Finally, the following corollary is derived from the fact that if a mechanism is Pareto dominated by a Pareto-efficient mechanism, it is also Pareto dominated by that mechanism whenever stable improvement is possible.

Corollary 1. There exists no strategy-proof and Pareto-efficient mechanism that Pareto dominates DAPL.

\subsection{Comparison with MSDA by Fragiadakis et al. (2015)}

Among others, DAPL most resembles the multistage DA mechanism (MSDA) by Fragiadakis et al. (2015). The common feature is that both mechanisms use precedence lists to control the set of students who propose to schools. Since MSDA is defined for problems without the types of students, let us focus on problems with $T=\left\{t_{1}\right\}$ in this subsection.

\section{Multi-stage DA}

Fix an arbitrary precedence list $r$ over all students. Start by setting $R^{0}=S, \underline{q}_{c}^{1}=\underline{q}_{c}^{t_{1}}$, $\bar{q}_{c}^{1}=\bar{q}_{c}^{t_{1}}$ for all $c \in C$. Let $n^{1}=\sum_{c \in C} \underline{q}_{c}^{1}$.

In stage $l \geq 1$ : 
1. Set $R^{l}=\left\{s \in S||\left\{s^{\prime} \in S \mid r(s)<r\left(s^{\prime}\right)\right\} \mid<n^{l}\right\}$, i.e., $R^{l}$ is the set of $n^{l}$ students with the lowest rankings according to $r$.

(a) If $R^{l-1} \backslash R^{l} \neq \emptyset$, run the standard DA mechanism for the students in $R^{l-1} \backslash R^{l}$ with maximum quotas $\left(\bar{q}_{c}^{l}\right)_{c \in C}$ for schools.

(b) If $R^{l-1} \backslash R^{l}=\emptyset$, run the standard DA mechanism for the students in $R^{l-1}$ with maximum quotas $\left(\underline{q}_{c}^{l}\right)_{c \in C}$ for schools.

2. Let $\mu^{l}$ be the assignment (for a subset of students) determined in step 1 of stage $l$. Remove all students who are assigned under $\mu^{l}$ from the market. If all students are assigned a school, end the algorithm. If not, proceed to step 3.

3. Define new quotas for each school $c \in C$ :

(a) $\bar{q}_{c}^{l+1}=\bar{q}_{c}^{l}-\left|\mu^{l}(c)\right|$.

(b) $\underline{q}_{c}^{l+1}=\max \left\{0, \underline{q}_{c}^{l}-\left|\mu^{l}(c)\right|\right\}$.

(c) $n^{l+1}=\sum_{c \in C} \underline{q}_{c}^{l+1}$.

4. Move to stage $l+1$.

Once the algorithm is completed (say after stage $L$ ), the final output of MSDA is the assignment in which the set of students assigned to school $c$ is $\cup_{l=1}^{L} \mu^{l}(c)$.

Despite the similarity between these two mechanisms, it turns out that MSDA does not achieve two of our main goals. That is, (i) MSDA is not strictly PL-fair, and (ii) MSDA may not be fair for a problem with B-common priority. And indeed, these negative observations are true for any choice of precedence lists to define MSDA.

Observation 2. MSDA (with any precedence lists) is not strictly PL-fair.

Observation 3. There is a problem with B-common priority for which MSDA (with any precedence lists) is not fair.

The following single example explains both of these two observations.

Example 5. Consider the following problem: $C=\left\{c_{1}, c_{2}, c_{3}\right\}, S=\left\{s_{1}, s_{2}, s_{3}\right\}$ and $T=\left\{t_{1}\right\}$. Priorities, capacities and type-specific constraints are summarized in the table below. 


\begin{tabular}{cccc} 
& $\succ_{c_{1}}$ & $\succ_{c_{2}}$ & $\succ_{c_{3}}$ \\
\hline & $s_{1}$ & $s_{1}$ & $s_{2}$ \\
& $s_{2}$ & $s_{2}$ & $s_{1}$ \\
& $s_{3}$ & $s_{3}$ & $s_{3}$ \\
capacity (ceiling for type $\left.t_{1}\right)$ & 2 & 1 & 1 \\
floor for type $t_{1}$ & 0 & 1 & 1 \\
\hline
\end{tabular}

Note that $\succ_{C}$ has B-common priority because $Q_{t_{1}}=1$ and $s_{3}$ has the lowest priority for all schools. Since $\left|S_{t_{1}}\right|=3$, there are six precedence lists to define MSDA.

First, consider a precedence list with $r_{1}: s_{1}, s_{2}, s_{3}$. And consider preferences $P_{S}$ such that $c_{3} P_{s} c_{2} P_{s} c_{1}$ for $s \in\left\{s_{1}, s_{2}\right\}$. In the first stage of MSDA with $r_{1}$, only $s_{1}$ can be in the DA mechanism because the sum of all floors is two. $s_{1}$ is assigned a slot of $c_{3}$, which is finalized, and the outcome is

$$
\mu=\left(\begin{array}{lll}
c_{1} & c_{2} & c_{3} \\
s_{3} & s_{2} & s_{1}
\end{array}\right)
$$

But this is not strictly PL-fair because $s_{2}$, who is not the bottom $Q_{t_{1}}$ student, PL-envies $s_{1}$ at $c_{3}$.

Next, consider a precedence list with $r_{2}: s_{2}, s_{1}, s_{3}$. The logic is the same as above: consider preferences $P_{S}^{\prime}$ such that $c_{2} P_{s}^{\prime} c_{3} P_{s}^{\prime} c_{1}$ for $s \in\left\{s_{1}, s_{2}\right\}$. Then the outcome of MSDA with $r_{2}$ is $\mu$, but this is not strictly PL-fair because $s_{1}$ PL-envies $s_{2}$ at $c_{2}$.

Finally, consider other four precedence lists $\left(r_{i}\right)_{i=3}^{6}$ such that $r_{i}\left(s_{3}\right) \in\{1,2\}$. Consider preferences $P_{S}^{\prime \prime}$ such that $c_{3} P_{s}^{\prime \prime} c_{2} P_{s}^{\prime \prime} c_{1}$ for all $s \in S$. In the outcome of MSDA with any $r_{i}$ $(i=3, \ldots, 6), s_{3}$ is assigned to either $c_{2}$ or $c_{3}$. But either $s_{1}$ or $s_{2}$ must be assigned to $c_{1}$, and the one who is assigned to $c_{1}$ PL-envies $s_{3}$. Thus, the outcome of MSDA is not strictly PL-fair.

In MSDA, bottom $n^{l}$ students according to $r$ cannot participate in DA in stage $l$ unless $l$ is the final stage, and the assignment is finalized in every stage. In addition, $n^{l}$ can be larger than $Q_{t_{1}}\left(n^{1}=2>1=Q_{t_{1}}\right)$ by definition. Therefore, when student $s_{2}$, who is not the bottom student under $r_{1}$, proposes to schools in MSDA, $s_{1}$ 's assignment is already finalized and this causes a justified envy. On the other hand, in DAPL, the assignment is not finalized until the DA stage terminates and this eliminates justified envy among all students who propose to school divisions.

Fragiadakis et al. (2015) show that MSDA is feasible, PL-fair, non-wasteful and group strategy-proof. Thus, DAPL improves MSDA with respect to fairness (without sacrificing 
other good properties), and it also accommodates separate floors and ceilings for different types.

\subsection{Maximal Domain Theorem}

We showed that the existence of fair and non-wasteful assignments is guaranteed for problems with B-common priority. But some real-life problems are unlikely to satisfy this condition, and we still need to know what will happen on these occasions. That is, can we still find stable assignments without B-common priority?

For this question, we take the following approach: given a primitive problem $\mathcal{Z}$, consider the maximal domain of a pair of two schools' strict rankings $\left(r_{c}^{t}, r_{c^{\prime}}^{t}\right)$ over type- $t$ students for the existence of fair and non-wasteful assignments. To do so, we consider a pairwise and type-specific version of the B-common priority condition. We say that $\succ_{c}$ is consistent with $r^{t}$ if $s \succ_{c} s^{\prime} \Leftrightarrow r^{t}(s)<r^{t}\left(s^{\prime}\right)$ for any $s, s^{\prime} \in S_{t}$.

Definition 8. A pair of strict rankings $\left(r_{c}^{t}, r_{c^{\prime}}^{t}\right)$ of two schools $c$ and $c^{\prime}$ over type- $t$ students has pairwise common priority among the bottom students (pairwise B-common priority) for $t \in T$ if

$$
B_{t}\left(r_{c}^{t}, K\right)=B_{t}\left(r_{c^{\prime}}^{t}, K\right)
$$

for any $K \in\left\{0, \ldots, Q_{t}\left(c, c^{\prime}\right)\right\}$.

We use the same threshold $Q_{t}\left(c, c^{\prime}\right)$ as B-common priority to define the set of type- $t$ bottom students between schools $c$ and $c^{\prime}$. Therefore, if $\succ_{C}$ has B-common priority, for any pair of priority rankings $\left(\succ_{c}, \succ_{c^{\prime}}\right)$ and any type $t$, the pair of strict rankings $\left(r_{c}^{t}, r_{c^{\prime}}^{t}\right)$ over type- $t$ students which is consistent with $\left(\succ_{c}, \succ_{c^{\prime}}\right)$ should also have pairwise B-common priority. ${ }^{17}$

Our next theorem shows that the maximal domain of $\left(r_{c}^{t}, r_{c^{\prime}}^{t}\right)$ is characterized by this pairwise B-common priority condition. More precisely, we show that for any $\left(r_{c}^{t}, r_{c^{\prime}}^{t}\right)$ which does not have pairwise B-common priority, there always exist $P_{S}$ and $\succ_{C}$ such that $\left(\succ_{c}, \succ_{c^{\prime}}\right)$ is consistent with $\left(r_{c}^{t}, r_{c^{\prime}}^{t}\right)$ and no fair and non-wasteful assignment exists. In particular, we can find $\succ_{C}$ such that common priority is only violated for $t$ and $\left(c, c^{\prime}\right)$.

For this result, we only consider primitive problems $\mathcal{Z}$ with the following condition.

\footnotetext{
${ }^{17}$ But the other direction is not straightforward because B-common priority requires the priority ranking of every school to be consistent with a single ranking $r^{t}$ for bottom students. It is still an open question whether we can always find a single ranking $r^{t}$ in the definition of B-common priority from the collection of pairwise B-common priority conditions for all pairs of schools and all types.
} 
Assumption 1. (Flexibility of primitive problems) Consider any nonempty $\tilde{C} \subseteq C$ with $|\tilde{C}| \leq m-2$ and $t \in T$ such that $F(\tilde{C}, t) \neq \emptyset$. For any $\left\{c, c^{\prime}\right\} \subseteq C \backslash \tilde{C}$, there exist $\mu, \mu^{\prime} \in F(\tilde{C}, t)$ which satisfy the following conditions:

1. there exists $s \in S_{t}$ such that $\mu(s)=c$ and $\mu^{\prime}(s)=c^{\prime}$;

2. there is at most one student $s^{\prime} \in S \backslash S_{t}$ such that $\mu\left(s^{\prime}\right)=c^{\prime}$ and $\mu^{\prime}\left(s^{\prime}\right)=c$; and

3. for any $s^{\prime \prime} \in S \backslash\left\{s, s^{\prime}\right\}, \mu\left(s^{\prime \prime}\right)=\mu^{\prime}\left(s^{\prime \prime}\right)$.

The flexibility condition ensures that whenever $F(\tilde{C}, t)$ is nonempty and there are two schools $c$ and $c^{\prime}$ in $C \backslash \tilde{C}$, one type- $t$ student $s$ can be assigned to either $c$ or $c^{\prime}$ under feasible assignments in $F(\tilde{C}, t) .{ }^{18}$ Moreover, when $s$ moves between the two slots at $c$ and $c^{\prime}$ under $\mu$ and $\mu^{\prime}$, there is at most one student $s^{\prime}$ who is affected by $s^{\prime}$ s move and other students' slots are kept unchanged. This condition is trivially satisfied when the capacities and maximum quotas of schools are large enough.

For flexible primitive problems, we establish the following maximal domain result.

Theorem 3. Consider any primitive problem $\mathcal{Z}$ that satisfies Assumption 1. For any pair of two schools' strict rankings $\left(r_{c}^{t}, r_{c^{\prime}}^{t}\right)$ over type-t students which does not have pairwise B-common priority, there exist

1. a preference profile of students $P_{S}$, and

2. a priority profile of schools $\succ_{C}$ such that

(a) $\left(\succ_{c}, \succ_{c^{\prime}}\right)$ is consistent with $\left(r_{c}^{t}, r_{c^{\prime}}^{t}\right)$;

(b) for any $c^{\prime \prime} \in C \backslash\left\{c, c^{\prime}\right\},\left(\succ_{c}, \succ_{c^{\prime \prime}}\right)$ has a common priority order for type $t$; and

(c) for any $(\tilde{c}, \hat{c}) \in C^{2}$ with $\tilde{c} \neq \hat{c},\left(\succ_{\tilde{c}}, \succ_{\hat{c}}\right)$ has a common priority order for any other type $t^{\prime} \in T \backslash\{t\}$

for which there exists no feasible, fair and non-wasteful assignment.

This theorem implies that if pairwise B-common priority is violated for just one type $t$ and one pair $\left(c, c^{\prime}\right)$ of schools, we can find a priority profile of schools which does not accommodate stable assignments for some student preferences. In our counterexample, the violation of stability can be due to the violation of non-wastefulness, fairness for same types

\footnotetext{
${ }^{18}$ This requires $\bar{q}_{c}^{t}>\underline{q}_{c}^{t}$ and $\bar{q}_{c^{\prime}}^{t}>\underline{q}_{c^{\prime}}^{t}$.
} 
or fairness across different types. ${ }^{19}$ We also require any pair of rankings to have a common priority order except for $t$ and $\left(c, c^{\prime}\right)$ (conditions 2(b) and 2(c)). This is because otherwise the non-existence problem can be easily caused by other parts of $\succ_{C}$, which makes our theorem trivial. This theorem complements the argument of our sufficiency theorem in the sense that the maximal domain of $\left(r_{c}^{t}, r_{c^{\prime}}^{t}\right)$ is characterized by a component of the sufficient condition, B-common priority.

One limitation of this theorem is that it does not identify the maximal domain of schools' entire priority rankings. Indeed, depending on how different types of students are ranked, there are priorities over all students for which the B-common priority condition is violated but a stable assignment still always exists. See Example 8 in Appendix D for details. It would be interesting to consider the maximal domain of schools' priority rankings over all students in future research.

\section{Application: Student-supervisor Matching}

As we mentioned in the Introduction, one of the interesting applications of our model is the student-supervisor matching problem in universities. In this section, we will explicate this problem further and examine the implication of B-common priority in the case study of Kawagoe and Matsubae (2017).

In Future University Hakodate in Japan, third-year students need to choose their supervisors to write their senior theses, and the university runs a centralized mechanism to assign students to supervisors. In addition to the maximum capacity, minimum quotas are set for supervisors because they need manpower for their laboratories and the university wants to achieve an equal share of supervision. In 2016, there were 254 students and 67 supervisors, which means that each supervisor was assigned roughly four students on average.

In this application, constraints have a special structure: supervisors have minimum quotas only for students who belong to the same course as they do. Here, a "course" is defined by a field of study. There are four courses for students (Complex Systems, Intelligent Systems, Information Systems and Information Design) and each student belongs to one of them. Each supervisor belongs to one of these four courses or Communication Media Laboratory (CML). Supervisors in the above-mentioned four courses have "same-course" minimum quotas, but there is no minimum quota for students in other courses. ${ }^{20}$ We denote the set

\footnotetext{
${ }^{19}$ This means that Theorem 3 cannot be easily strengthened to the non-existence of assignments with only one or two of these three properties.

${ }^{20}$ This means that supervisors in CML do not have any minimum quotas.
} 
of type- $t$ supervisors by $C_{t}$. In 2016, the total capacity for each supervisor was 6 if he is in Complex Systems or Intelligent Systems, and 4 otherwise. The minimum quota for the same-course students was 2 for supervisors in Complex Systems, Intelligent Systems or Information Systems, and 3 for those in Information Design. These numbers are summarized in Table 1.

\begin{tabular}{c||c|c|c|c|c|} 
course $(t)$ & $\begin{array}{c}\text { Complex } \\
\text { Systems }\end{array}$ & $\begin{array}{c}\text { Intelligent } \\
\text { Systems }\end{array}$ & $\begin{array}{c}\text { Information } \\
\text { Systems }\end{array}$ & $\begin{array}{c}\text { Information } \\
\text { Design }\end{array}$ & CML \\
\hline \hline \# of students $\left(\left|S_{t}\right|\right)$ & 62 & 62 & 86 & 44 & 0 \\
\# of supervisors $\left(\left|C_{t}\right|\right)$ & 13 & 12 & 19 & 13 & 10 \\
capacity $\left(q_{c}\right.$ for $\left.c \in C_{t}\right)$ & 6 & 6 & 4 & 4 & 4 \\
minimum quota $\left(\underline{q}_{c}^{t}\right.$ for $\left.c \in C_{t}\right)$ & 2 & 2 & 2 & 3 & 0 \\
$Q_{t}(c)$ for $c \in C \backslash C_{t}$ & 26 & 24 & 38 & 39 & 0 \\
$Q_{t}(c) / S_{t}$ & 0.42 & 0.39 & 0.44 & 0.89 & 0
\end{tabular}

Table 1: Case of Future University Hakodate in 2016 (Kawagoe and Matsubae, 2017)

Now, let us discuss the applicability and implication of our B-common priority in this problem. First, it is possible for the university to adopt a common ranking of students (such as GPAs) in this context because supervisors do not have veto power on this decision. Second, by requiring only B-common priority (rather than common priority), we could give supervisors freedom to rank many good students as they like. According to the data from 2016, we can easily compute $Q_{t}(c)$ for each course $t$ and each supervisor $c{ }^{21}$ Table 1 shows $Q_{t}(c)$ for each course $t$ and supervisors $c \in C \backslash C_{t}$ as this is the maximal number of type- $t$ students who should be ranked in the same way by some two supervisors under B-common priority. For example, $Q_{t}(c)$ for Complex Systems $(t)$ and some supervisor who is not in Complex Systems $\left(c \in C \backslash C_{t}\right)$ is simply the sum of the minimum quotas for type- $t$ students, that is, $2 \times 13=26$. The percentage $Q_{t}(c) / S_{t}$ of the bottom students in course $t$ is around $40 \%$ for Complex Systems, Intelligent Systems and Information Systems, and $89 \%$ for Information Design. This means that for students in each of the first three courses, we can ask supervisors to rank around $40 \%$ of them according to their GPAs, and let them rank the rest of $60 \%$ as they like. But there is much less freedom to rank students in Information Design because of the high minimum quotas for this course.

\footnotetext{
${ }^{21}$ This is because the capacities are large enough to find an assignment in which all minimum quotas are binding.
} 


\section{Concluding Remarks}

This paper identified the restrictions on the schools' priority structure to guarantee the existence of stable assignments when there are type-specific minimum quotas. First, we showed that for any problem with B-common priority, DAPL always finds a fair and nonwasteful assignment. DAPL has good properties in general: feasibility, non-wastefulness, strict PL-fairness, and group strategy-proofness. DAPL is also shown to satisfy good efficiency properties as well. Next, we showed a weak form of necessity theorem: pairwise B-common priority characterizes the maximal domain of two schools' priority rankings for the existence of stable assignments for any flexible primitive problem.

Our maximal domain theorem is derived for the domain of two schools' priority rankings over each type of students, not over all students. Therefore we leave the maximal domain theorem of schools' entire priority rankings for future research.

Throughout the paper, we discussed several important applications of our model. Our existence result is relevant to problems such as the student-supervisor matching in which B-common priority may be adopted. But DAPL might serve as a new solution for other

problems without B-common priority. Thus, it will be interesting to study more real-world matching markets with type-specific minimum quotas and further examine the implication of our results.

\section{References}

AbdulkadiroĞLu, A. (2005): "College Admission with Affirmative Action," International Journal of Game Theory, 33, 535-549.

AbdulkadiroĞlu, A., P. A. Pathak, And A. E. Roth (2005): "The New York City High School Match," American Economic Review, Papers and Proceedings, 95, 364-367.

AbdulkadiroĞLu, A. And T. Sönmez (2003): "School Choice: A Mechanism Design Approach," American Economic Review, 93, 729-747.

Aygün, O. And I. Bó (2016): "College Admission with Multidimensional Privileges: The Brazilian Affirmative Action Case," Unpublished manuscript.

Aygün, O. And B. Turhan (2016): "Dynamic Reserves in Matching Markets: Theory and Applications," Unpublished manuscript. 
Balinski, M. And T. Sönmez (1999): "A Tale of Two Mechanisms: Student Placement," Journal of Economic Theory, 84, 73-94.

Biró, P., T. Fleiner, R. W. Irving, and D. F. Manlove (2010): "College Admissions Problem with Lower and Common Quotas," Theoretical Computer Science, 411, 31363153.

Bó, I. (2016): "Fair Implementation of Diversity in School Choice," Games and Economic Behavior, 97, 54-63.

Delacrétaz, D., S. D. Kominers, and A. Teytelboym (2016): "Refugee Resettlement," Unpublished manuscript.

DoĞAN, B. (2016): "Responsive Affirmative Action in School Choice," Journal of Economic Theory, 165, 69-105.

Dur, U., S. D. Kominers, P. Pathak, and T. Sönmez (forthcoming): "Reserve Design: Unintended Consequences and the Demise of Boston's Walk Zones," Journal of Political Economy.

Dur, U., P. Pathak, And T. Sönmez (2017): "Explicit vs. Statistical Targeting in Affirmative Action: Theory and Evidence from Chicago's Exam Schools," NBER Working Paper.

Echenique, F. And M. B. Yenmez (2015): "How to Control Controlled School Choice," American Economic Review, 105, 2679-2694.

Ehlers, L. (2010): "School Choice with Control," CIREQ working paper.

Ehlers, L., I. E. Hafalir, M. B. Yenmez, And M. A. Yildirim (2014): "School Choice with Controlled Choice Constraints: Hard Bounds versus Soft Bounds," Journal of Economic Theory, 153, 648-683.

Erdil, A. And T. Kumano (2012): "Prioritizing Diversity in School Choice," Unpublished manuscript.

Ergin, H. (2002): "Efficient Resource Allocation on the Basis of Priorities," Econometrica, 70, 2489-2497. 
Fragiadakis, D. E., A. Iwasaki, P. Troyan, S. Ueda, and M. Yokoo (2015): "Strategyproof Matching with Minimum Quotas," ACM Transactions on Economics and Computation, 4, Article 6.

Fragiadakis, D. E. And P. Troyan (2017): "Improving Matching under Hard Distributional Constraints," Theoretical Economics, 12, 863-908.

Goto, M., A. Iwasaki, Y. Kawasaki, R. Kurata, Y. Yasuda, and M. Yokoo (2016): "Strategyproof Matching with Regional Minimum and Maximum Quotas," Artificial Intelligence, 235, 40-57.

Goto, M., F. Kojima, R. Kurata, A. Tamura, and M. Yokoo (2017): "Designing Matching Mechanisms under General Distributional Constraints," American Economic Journal: Microeconomics, 9, 226-262.

Haeringer, G. AND F. Klijn (2009): "Constrained School Choice," Journal of Economic Theory, 144, 1921-1947.

Hafalir, I. E., M. B. Yenmez, and F. Kojima (2017): "Integrating School Districts: Diversity, Balance, and Welfare," Unpublished manuscript.

Hafalir, I. E., M. B. Yenmez, And M. A. Yildirim (2013): "Effective Affirmative Action in School Choice," Theoretical Economics, 8, 325-363.

Hatfield, J. W. and F. Kojima (2009): "Group Incentive Compatibility for Matching with Contracts," Games and Economic Behavior, 67, 745-749.

Kamada, Y. And F. KoJima (2015): "Efficient Matching under Distributional Constraints: Theory and Applications," American Economic Review, 105, 67-99.

_ (2017): "Stability Concepts in Matching under Distributional Constraints," Journal of Economic Theory, 168, 107-142.

_ (forthcoming): "Stability and Strategy-proofness for Matching with Constraints: A Necessary and Sufficient Condition," Theoretical Economics.

Kawagoe, T. and T. Matsubae (2017): "Matching with Minimal Quota: A Case Study of the Japanese University Student-Supervisor Assignment," Unpublished manuscript.

Kesten, O. (2006): "On Two Competing Mechanisms for Priority-based Allocation Problems," Journal Economic Theory, 127, 155-171. 
- (2010): "School Choice with Consent," Quarterly Journal of Economics, 125, 12971348.

Kojima, F. (2012): "School Choice: Impossibilities for Affirmative Action," Games and Economic Behavior, 75, 685-693.

Kominers, S. D. And T. SÖnmez (2016): "Matching with Slot-Specific Priorities: Theory," Theoretical Economics, 11, 683-710.

Kumano, T. (2013): "Strategy-proofness and Stability of the Boston Mechanism: An Almost Impossibility Result," Journal of Public Economics, 105, 23-29.

Kurata, R., N. Hamada, C.-L. Hsu, T. Suzuki, S. Ueda, and M. Yokoo (2016): "Pareto Efficient Strategy-proof School Choice Mechanism with Minimum Quotas and Initial Endowments," Proceedings of the 15th International Conference on Autonomous Agents and Multiagent Systems, 59-67.

Westkamp, A. (2013): "An Analysis of the German University Admissions System," Economic Theory, 53, 561-589. 


\section{Appendix A Lemmas}

Lemma 1 and Lemma 2 are used in the first part (feasibility) of Proposition 1 and Proposition 2 .

Lemma 1. Consider DAPL for any $\left(\mathcal{Z}, \succ_{C}, P_{S}\right)$. For each type $t \in T$, there are at least two schools whose type-t divisions are filled only by real students in the DA stage. (If the quota of a school's type-t division is zero, we say that this quota is filled by real students.)

Proof: First, since we have $\left|S_{t}\right|>\sum_{c \in C} \underline{q}_{c}^{t}$, in steps of DAPL where no type- $t$ division is filled only by real students, at least one artificial student is rejected. And this artificial student proposes to the type- $t$ null division in the next step. Repeating this, at least one school's type- $t$ division must be filled by real students.

Second, by additional rejection steps of DAPL, if there is only one school whose type- $t$ division is filled by real students, one type- $t$ artificial student is rejected from $c_{m}^{t}$ or $c_{m-1}^{t}$ and she proposes to the type- $t$ null division. And in the next step, one real type- $t$ student proposes to some school division. Since each open division $c^{o}$ can accept at most $\min \left\{\bar{q}_{c}^{t}-\right.$ $\left.\underline{q}_{c}^{t},\left|S_{t}\right|-\sum_{c^{\prime} \in C} \underline{q}_{c^{\prime}}^{t}\right\}$ type-t students, when the DA stage terminates, at least two schools' type- $t$ divisions are filled by real students.

Lemma 2. Consider DAPL for any $\left(\mathcal{Z}, \succ_{C}, P_{S}\right)$. Suppose that there are two schools $c_{1}$ and $c_{2}$ whose type-t divisions are filled only by real students in the DA stage. Let $C_{b} \subseteq C \backslash\left\{c_{1}, c_{2}\right\}$ be the set of schools $c$ such that $\left|\mu^{* t}(c)\right| \leq \underline{q}_{c}^{t}$ where $\mu^{*}$ is the outcome of DAPL for $\left(\mathcal{Z}, \succ_{C}, P_{S}\right)$. Then we have $\sum_{c \in C_{b}} \underline{q}_{c}^{t} \leq Q_{t}\left(c_{1}, c_{2}\right)$.

Proof: Suppose by contradiction that $\sum_{c \in C_{b}} \underline{q}_{c}^{t}>Q_{t}\left(c_{1}, c_{2}\right)$. If there is any school $c \in$ $C_{b}$ such that $\left|\mu^{* t}(c)\right|<\underline{q}_{c}^{t}$, we can move type- $t$ students from $C \backslash C_{b}$ to $C_{b}$ so that the unfilled minimum quotas of such schools in $C_{b}$ are exactly filled. This is possible without violating any other constraints. Since we can do this for every type, there is a feasible assignment under which the type- $t$ minimum quota $\underline{q}_{c}^{t}$ of any school $c \in C_{b}$ is binding. ${ }^{22}$ Then $\sum_{c \in C_{b}} \underline{q}_{c}^{t}>Q_{t}\left(c_{1}, c_{2}\right)$ is a contradiction to the definition of $Q_{t}\left(c_{1}, c_{2}\right)$, and we must have $\sum_{c \in C_{b}} \underline{q}_{c}^{t} \leq Q_{t}\left(c_{1}, c_{2}\right)$.

Lemma 3 is used in the fifth part (group strategy-proofness) of Proposition 1. Consider a variant of DAPL in which additional rejection steps are removed. We call this version of DAPL as DAPL without Additional Rejection Steps. As functions, we denote DAPL by $\gamma^{D A P L}$ and DAPL without Additional Rejection Steps by $\gamma^{D A P L w A R S}$.

\footnotetext{
${ }^{22}$ We can also find such a feasible assignment when there is no school $c \in C_{b}$ such that $\left|\mu^{* t}(c)\right|<\underline{q}_{c}^{t}$.
} 
Lemma 3. Consider DAPL for any $\left(\mathcal{Z}, \succ_{C}, P_{S}\right)$. Let $T^{*}$ be the set of types for which at least one artificial student is rejected in an additional rejection step. Also for each $t \in T^{*}$, let $c^{*}(t)$ be the unique school whose type-t division was filled only by real students in the additional rejection step. Then the following two statements hold.

1. For any $s \in S$ with $\tau(s) \in T \backslash T^{*}, \gamma_{s}^{D A P L}\left(\mathcal{Z}, \succ_{C}, P_{S}\right)=\gamma_{s}^{D A P L w A R S}\left(\mathcal{Z}, \succ_{C}, P_{S}\right)$.

2. For any $s \in S$ with $\tau(s) \in T^{*}$ who was assigned to a school division in the DA stage of DAPL, if there is $c \in C$ such that $c P_{s} \gamma_{s}^{D A P L}\left(\mathcal{Z}, \succ_{C}, P_{S}\right)$, then $c=c^{*}(t)$.

Proof: 1 . Consider any type $t \in T^{*}$. After any additional rejection step in which $a \in A_{t}$ is rejected, $a$ proposes to $\phi^{t}$ and one type- $t$ real student $s$ is rejected by $\phi^{t}$. If $s$ proposes to the type- $t$ division of $c \neq c^{*}(t)$ in the next step, $s$ is assigned to $c^{t}$ and type- $t$ artificial students fill the remaining slots of all type- $t$ divisions. If $s$ proposes to the open division of $c^{*}(t)$ in the next step and $s$ is tentatively accepted, another type- $t$ student $s^{\prime}$ should be rejected from it because the open division of $c^{*}(t)$ already accepts $\left|S_{t}\right|-\sum_{c^{\prime} \in C} \underline{q}_{c^{\prime}}^{t}$ type- $t$ students. And in the next step, $s^{\prime}$ will be accepted by a type- $t$ division of some school $c^{\prime} \neq c^{*}(t)$.

In either sequence of steps, no student of any other type is rejected following the additional rejection step for type $t$. Therefore, for any for any $\tilde{s} \in S$ with $\tau(\tilde{s}) \in T \backslash T^{*}, \gamma_{\tilde{s}}^{D A P L}\left(\mathcal{Z}, \succ_{C}\right.$ ,$\left.P_{S}\right)=\gamma_{\tilde{s}}^{D A P L w A R S}\left(\mathcal{Z}, \succ_{C}, P_{S}\right)$ should hold.

2. Consider an additional rejection step in which a type- $t$ artificial student is rejected. In this step, $c^{*}(t)$ is the only school whose type- $t$ division is fully filled by real students. This means that any student $s \in S_{t}$ can be assigned to the type-t division of any school in $C \backslash\left\{c^{*}(t)\right\}$ if she proposes to it in the DA stage. And this school becomes the final assignment of $s$ in DAPL. Therefore, if $s$ proposed to school divisions in the DA stage and prefers another school to her own assignment, then it must be $c^{*}(t)$.

\section{Appendix B Proofs of the Main Results}

As in Appendix A, we denote DAPL by $\gamma^{D A P L}$ and DAPL without Additional Rejection Steps by $\gamma^{D A P L w A R S}$.

\section{B.1 Proof of Proposition 1}

Take any problem $\left(\mathcal{Z}, \succ_{C}\right)$ and any profile of students' preferences $P_{S}$. Let $\mu^{*}$ be the assignment produced by DAPL for $\left(\mathcal{Z}, \succ_{C}, P_{S}\right)$. 


\section{Feasibility}

First, it is easy to show that every student is assigned to some school under $\mu^{*}$. Students are either assigned to the school divisions or the null divisions after the DA stage. Those who are in the school divisions are finalized to these slots, and those who are assigned to the null divisions are assigned the slots of minimum quotas in the MQ stage. Thus, every student is assigned a slot.

Next, we need to show that all capacity and type-specific constraints are satisfied under $\mu^{*}$. By the definition of DAPL, no total and type-specific maximum quota is violated under $\mu^{*}$. Then it suffices to show that for any $(t, c) \in T \times C$, the type- $t$ minimum quota of school $c$ is satisfied under $\mu^{*}$, i.e., $\left|\mu^{* t}(c)\right| \geq \underline{q}_{c}^{t}$. Consider an arbitrary type $t \in T$. Since at least two schools' type- $t$ divisions are filled by real students in the DA stage by Lemma 1, let $c_{1}$ and $c_{2}$ be such schools. Let $C_{b} \subseteq C \backslash\left\{c_{1}, c_{2}\right\}$ be the set of schools $c$ such that $\left|\mu^{* t}(c)\right| \leq \underline{q}_{c}^{t}$. By Lemma 2, we have $\sum_{c \in C_{b}} \underline{q}_{c}^{t} \leq Q_{t}\left(c_{1}, c_{2}\right)$. Note that for any school $c \in C \backslash C_{b}, c^{t}$ is fully filled by real students in the DA stage because we have $\left|\mu^{* t}(c)\right|>\underline{q}_{c}^{t}$ or $c \in\left\{c_{1}, c_{2}\right\}$. And since there are $Q_{t}\left(\geq Q_{t}\left(c_{1}, c_{2}\right) \geq \sum_{c \in C_{b}} \underline{q}_{c}^{t}\right)$ type- $t$ artificial students, $\sum_{c \in C_{b}} \underline{q}_{c}^{t}$ minimum quotas of $C_{b}$ must be filled by artificial students in the DA stage if they are not filled by real students. Thus, $\left|\mu^{* t}(c)\right|=\underline{q}_{c}^{t}$ for any $c \in C_{b}$, which means that all minimum quotas are filled under $\mu^{*}$.

\section{2-4. Non-wastefulness and strict PL-fairness}

Take any student $s \in S$ and let $t \equiv \tau(s)$. Consider any school $c \in C$ such that $c P_{s} \mu^{*}(s)$ (if there is any). It suffices to consider the following two cases.

[1] When $s$ is assigned a slot of the null division $\phi^{t}$ in the DA stage.

As the serial dictatorship is used to assign $s$ in the MQ stage, any type- $t$ student who is assigned to $c$ at $\mu^{*}$ should be ranked higher than $s$ in the precedence list $r^{t}$. In addition, $s$ is one of the bottom $Q_{t}$ students in the precedence list $r^{t}$ because she is assigned to $\phi^{t}$. It is obvious that the minimum quota of $\mu^{*}(s)$ for type- $t$ students is binding at $\mu^{*}$. Therefore, $s$ does not PL-envy same type students. Moreover, as the minimum quota of $\mu^{*}(s)$ for type- $t$ students is binding at $\mu^{*}, s$ does not justifiably envy students of other types. This also implies that $s$ cannot justifiably claim an empty slot of $c$ even if some of $c$ 's slots are not filled.

[2] When $s$ is assigned a slot of the type- $t$ division or the open division of $\mu^{*}(s)$ in the DA stage.

$s$ must have proposed to both divisions $c^{t}$ and $c^{o}$, and must have been rejected by both of them. This means that $s$ neither justifiably envies a student in $\mu^{*}(c)$ nor justifiably claims 
an empty slot of $c$.

5. Group strategy-proofness

Suppose by contradiction that for some $\left(\mathcal{Z}, \succ_{C}, P_{S}\right)$, there are $I \subseteq S$ and $P_{I}^{\prime}$ such that $\gamma_{s}^{D A P L}\left(\mathcal{Z}, \succ_{C}, P_{I}^{\prime}, P_{S \backslash I}\right) P_{s} \gamma_{s}^{D A P L}\left(\mathcal{Z}, \succ_{C}, P_{S}\right)$ for every $s \in I$.

Let $T^{*}$ be the set of types for which at least one artificial student is rejected in an additional rejection step of DAPL for $\left(\mathcal{Z}, \succ_{C}, P_{S}\right)$. Also for each $t \in T^{*}$, let $c^{*}(t)$ be the unique school whose type- $t$ division was filled only by real students in the additional rejection steps.

[1] $I \cap\left\{s \in S \mid \tau(s) \in T^{*}\right\}=\emptyset$.

By Lemma 3, the assignment of DAPL is the same as that of DAPL without Additional Rejection Steps for any students in $I$. That is, we have

$$
\gamma_{s}^{D A P L w A R S}\left(\mathcal{Z}, \succ_{C}, P_{I}^{\prime}, P_{S \backslash I}\right) P_{s} \gamma_{s}^{D A P L w A R S}\left(\mathcal{Z}, \succ_{C}, P_{S}\right)
$$

for every $s \in I$.

Now let $D \subseteq S$ be the set of students who are assigned to school divisions in the DA stage of DAPL without Additional Rejection Steps under $P_{S}$. We must have $I \cap D \neq \emptyset$ because students cannot get strictly better off by manipulating preferences only in the MQ stage.

Then, consider DAPL without Additional Rejection Steps with only students in $D$. In this problem, the assignment of each student $s \in D$ when $P_{D}$ is submitted is $\gamma_{s}^{D A P L w A R S}\left(\mathcal{Z}, \succ_{C}\right.$ ,$P_{S}$ ) by the definition of $D$. As there are less students in the market and school preferences are responsive, students in $I \cap D$ can still get strictly better off by submitting some $P_{I \cap D}^{\prime \prime}$ in this problem. Let us focus on the extended problem in the DA stage of this DAPL without Additional Rejection Steps under $\left(P_{I \cap D}^{\prime \prime}, P_{D \backslash I}\right)$. If a student in $I \cap D$ is assigned to a school division, then this is a strict improvement in her extended preference. Even if a student in $I \cap D$ is assigned to a null division, this is also a strict improvement in her extended preference as the null division is assumed to be the first choice for any real student. Therefore, $I \cap D$ can get strictly better off by manipulating their preferences in the extended problem of the DA stage as well. However, since this DA stage is equivalent to the DA mechanism with type-specific maximum quotas by Abdulkadiroğlu (2005), this is a contradiction to its group strategy-proofness, which is shown by Hatfield and Kojima (2009).

[2] $I \cap\left\{s \in S \mid \tau(s) \in T^{*}\right\} \neq \emptyset$.

By Lemma 3, for any $t \in T^{*}$ and $s \in S_{t}$, if $s$ is assigned to $c^{*}(t)$ in DAPL under $P_{S}, c^{*}(t)$ is the first choice for $s$. Thus, for any student $s \in I \cap\left\{s \in S \mid \tau(s) \in T^{*}\right\}$, one of the following 
two must be true: (i) $s$ is assigned to $c^{t}$ with $c \neq c^{*}(t)$ in the DA stage of DAPL under $P_{S}$; or (ii) $s$ is assigned to $\phi^{t}$ in the DA stage of DAPL under $P_{S}$.

First, let $I_{1} \subseteq I$ be the set of students of case (i). Note that any $s \in I_{1}$ must be assigned to $c^{*}(t)$ in DAPL under $\left(P_{I}^{\prime}, P_{S \backslash I}\right)$ because $c^{*}(t)$ is the only school which can strictly improve $s^{\prime}$ 's assignment by Lemma 3. Suppose that for each $s \in I_{1}, P_{s}^{\prime}$ is such that $c^{\prime} P_{s}^{\prime} \gamma_{s}^{D A P L}\left(\mathcal{Z}, \succ_{C}, P_{S}\right)$ $\Rightarrow c^{\prime}=c^{*}(t)$. Then, we would have $\gamma_{s}^{D A P L}\left(\mathcal{Z}, \succ_{C}, P_{I}^{\prime}, P_{S \backslash I}\right)=\gamma_{s}^{D A P L}\left(\mathcal{Z}, \succ_{C}, P_{S}\right)$ for any $s \in I_{1}$ since the DA stage gives the same assignment under either preferences, and this is a contradiction. Thus, there must be some $\tilde{s} \in I_{1}$ for whom there is $c^{\prime} \neq c^{*}(t)$ with $c^{\prime} P_{\tilde{s}}^{\prime} \gamma_{\tilde{s}}^{D A P L}\left(\mathcal{Z}, \succ_{C}, P_{S}\right)$. But then, since any type- $t$ division of a school in $C \backslash\left\{c^{*}(t)\right\}$ should have an artificial student or a vacant slot when students in $I_{1}$ propose to it in DAPL under $P_{S}$, some $\hat{s} \in I_{1}$ must be assigned to the type- $t$ division of $\hat{c} \neq c^{*}(t)$ in DAPL under $\left(P_{I}^{\prime}, P_{S \backslash I}\right)$. But this is a contradiction and we have $I_{1}=\emptyset$.

Then, any student in $I$ must be of case (ii). But since the DA stage of DAPL under $P_{S}$ and that of DAPL under $\left(P_{I}^{\prime}, P_{S \backslash I}\right)$ are exactly the same, students in $I$ cannot get strictly better off by manipulating preferences in the MQ stage. Thus, this is also a contradiction.

\section{B.2 Proof of Proposition 2}

Consider any $\left(\mathcal{Z}, \succ_{C}, P_{S}\right)$ with B-common priority, and any $(t, c) \in T \times C$. By the definition of the precedence list $r^{t}, B_{t}\left(\succ_{c}, K\right)=B_{t}\left(r^{t}, K\right)$ holds for any $K \in\left\{0, \ldots, Q_{t}(c)\right\}$.

To show that the outcome of DAPL is fair, it suffices to show that no student justifiably envies another student of the same type at $c$ when she is assigned to the type- $t$ null division $\phi^{t}$ at the end of the DA stage.

[1] When $c^{t}$ is fully filled by real students in the DA stage.

By Lemma 1, there exists another school $c^{\prime} \neq c$ whose type- $t$ division is filled only by real students in the DA stage. Then, there are at most $Q_{t}\left(c, c^{\prime}\right)$ type- $t$ minimum quotas in $C \backslash\left\{c, c^{\prime}\right\}$ that are binding at a feasible assignment. This implies that there are at most $Q_{t}\left(c, c^{\prime}\right)$ real students who are finally assigned to $\phi^{t}$ in the DA stage. Since these real students are in $B_{t}\left(\succ_{c}, Q_{t}(c)\right)$, they cannot justifiably envy type- $t$ students assigned to $c$.

[2] When $c^{t}$ is not fully filled by real students in the DA stage.

Let $\tilde{C} \subseteq C$ be the set of schools such that $|\tilde{C}| \leq m-2$ and $\sum_{c^{\prime \prime} \in \tilde{C}} \underline{q}_{c^{\prime \prime}}^{t}=Q_{t}$. If $c \notin \tilde{C}$, there is another school $c^{\prime} \notin \tilde{C}$ and we have $Q_{t}=Q_{t}\left(c, c^{\prime}\right)$. If $c \in \tilde{C}$, take a school $c^{\prime} \notin \tilde{C}$ and $Q_{t}-\underline{q}_{c}^{t} \leq Q_{t}\left(c, c^{\prime}\right)$ holds. Therefore, we have $Q_{t}-\underline{q}_{c}^{t} \leq Q_{t}(c)$ in either case, and thus, $Q_{t}-Q_{t}(c) \leq \underline{q}_{c}^{t}$. 
Let $S_{t}^{1} \equiv B_{t}\left(r^{t}, Q_{t}(c)\right)$ be the bottom $Q_{t}(c)$ students in the ranking $r^{t}$, and let $S_{t}^{2} \equiv$ $B_{t}\left(r^{t}, Q_{t}\right) \backslash B_{t}\left(r^{t}, Q_{t}(c)\right)$ be the next bottom $Q_{t}-Q_{t}(c)$ students in the ranking $r^{t}$.

First, it is clear that no student in $S_{t}^{1}$ justifiably envies another type- $t$ student who is assigned to $c$ because $\succ_{c}$ is consistent with $r^{t}$ for students in $S_{t}^{1}$.

Next, consider $S_{t}^{2}$. Since common priority is only applied to the bottom $Q_{t}(c)$ students, students in $S_{t}^{2}$ may have high priority in $\succ_{c}$. And students in $S_{t}^{2}$ may be assigned to $\phi^{t}$ in the DA stage. Now we show that even if a student in $S_{t}^{2}$ is assigned to $\phi^{t}$, she can be assigned to $c$ in the MQ stage if she prefers it, and therefore she does not envy type- $t$ students assigned to $c$.

Since $c^{t}$ is not filled by real students in the DA stage, we can take two schools $c_{1}$ and $c_{2}$ from $C \backslash\{c\}$ whose type- $t$ divisions are filled only by real students in the DA stage by Lemma 1. And by Lemma 2, we have $\sum_{c \in C_{b}} \underline{q}_{c}^{t} \leq Q_{t}\left(c_{1}, c_{2}\right) \leq Q_{t}$ where $C_{b} \subseteq C \backslash\left\{c_{1}, c_{2}\right\}$ is the set of schools whose type- $t$ divisions were not filled by real students in the DA stage. We have $c \in C_{b}$ by definition. Now let $n_{t}^{c}$ be the number of type- $t$ real students who are assigned to $c^{t}$ in the DA stage, and let $n_{t}$ be the number of type- $t$ artificial students who are assigned to $\phi^{t}$ in the DA stage. At the end of the DA stage, since there are at most $\sum_{c \in C_{b}} \underline{q}_{c}^{t}-n_{t}^{c}$ type- $t$ artificial students who are assigned to school divisions, there are at least $Q_{t}-\left(\sum_{c \in C_{b}} \underline{q}_{c}^{t}-n_{t}^{c}\right)$ type- $t$ artificial students who are assigned to $\phi^{t}$ in the DA stage. This implies $n_{t} \geq Q_{t}-\left(\sum_{c \in C_{b}} \underline{q}_{c}^{t}-n_{t}^{c}\right) \geq n_{t}^{c}$.

Since we have $\left|S_{t}^{2}\right| \leq \underline{q}_{c}^{t}$ from the discussion above,

$$
\max \left\{0,\left|S_{t}^{2}\right|-n_{t}\right\} \leq \underline{q}_{c}^{t}-n_{t}^{c}
$$

holds. This means that the number of type-t minimum quota of $c$ which need to be filled in the MQ stage $\left(\underline{q}_{c}^{t}-n_{t}^{c}\right)$ is weakly greater than the number of students in $S_{t}^{2}$ who are assigned to $\phi^{t}\left(\max \left\{0,\left|S_{t}^{2}\right|-n_{t}\right\}\right)$. Therefore, even if a student in $S_{t}^{2}$ is assigned to $\phi^{t}$, she could always be assigned to $c$ in the MQ stage if she prefers it. This implies that no student in $S_{t}^{2}$ justifiably envies a student of type $t$ who is assigned to $c$ in DAPL.

\section{B.3 Proof of Proposition 3}

Suppose by contradiction that there is $\left(\mathcal{Z}, \succ_{C}, P_{S}\right)$ such that $\gamma^{D A P L}\left(\mathcal{Z}, \succ_{C}, P_{S}\right)$ is Pareto dominated by a feasible, fair and non-wasteful assignment $\mu$. Then, let $I \subseteq S$ be the set of students whose assignments are strictly improved under $\mu$, i.e., $\mu(s) P_{s} \gamma_{s}^{D A P L}\left(\mathcal{Z}, \succ_{C}, P_{S}\right)$ for all $s \in I$; and $\mu(s)=\gamma_{s}^{D A P L}\left(\mathcal{Z}, \succ_{C}, P_{S}\right)$ for all $s \in S \backslash I$. Let $D \subseteq S$ be the set of students who are assigned to school divisions in the DA stage of DAPL. There are three possibilities: [1] $I \subseteq S \backslash D,[2] I \subseteq D$, and [3] $I \cap(S \backslash D) \neq \emptyset$ and $I \cap D \neq \emptyset$, 
$[1] I \subseteq S \backslash D$.

The assignments of students in $S \backslash D$ are determined by the serial dictatorship in the MQ stage. Since $\gamma^{D A P L}\left(\mathcal{Z}, \succ_{C}, P_{S}\right)$ is non-wasteful, if all students in $I$ are strictly better off under $\mu$, some students in $S \backslash I$ must be worse off under $\mu$. But this is a contradiction.

[2] $I \subseteq D$.

Consider the extended problem in the DA stage. By $|T|=1$, any justified envy among students in $D$ in this extended problem corresponds to a justified envy in the original problem. Since $\gamma^{D A P L}\left(\mathcal{Z}, \succ_{C}, P_{S}\right)$ is Pareto dominated by $\mu$, which is feasible, fair and non-wasteful, there must be a corresponding feasible, fair and non-wasteful assignment in the extended problem which Pareto improves the assignment of DAPL in the DA stage. But this contradicts to the fact that the standard DA mechanism is student-optimal among all feasible, fair and non-wasteful assignments.

[3] $I \cap(S \backslash D) \neq \emptyset$ and $I \cap D \neq \emptyset$.

Since $\gamma^{D A P L}\left(\mathcal{Z}, \succ_{C}, P_{S}\right)$ is non-wasteful, $\mu$ must be achieved by students in $I$ exchanging their slots under $\gamma^{D A P L}\left(\mathcal{Z}, \succ_{C}, P_{S}\right)$. And since [1] and [2] do not hold, there must be a student $s \in I \cap D$ and $s^{\prime} \in I \cap(S \backslash D)$ such that $\mu(s)=\gamma_{s^{\prime}}^{D A P L}\left(\mathcal{Z}, \succ_{C}, P_{S}\right)$. But this is a contradiction because $s$ should have proposed to $\gamma_{s^{\prime}}^{D A P L}\left(\mathcal{Z}, \succ_{C}, P_{S}\right)$ in the DA stage and should have been assigned to it.

\section{B.4 Proof of Proposition 4}

Consider the same problem $\left(\mathcal{Z}, \succ_{C}\right)$ as in Example 4. $C=\left\{c_{1}, c_{2}, c_{3}, c_{4}\right\}, S=\left\{s_{1}, s_{2}, s_{3}, s_{4}\right\}$, $T=\left\{t_{1}, t_{2}\right\}, \tau\left(s_{1}\right)=\tau\left(s_{2}\right)=t_{1}$, and $\tau\left(s_{3}\right)=\tau\left(s_{4}\right)=t_{2}$. Priorities, preferences of interest, capacities and type-specific constraints are summarized in the table below.

\begin{tabular}{cccccccccc} 
& $\succ_{c_{1}}$ & $\succ_{c_{2}}$ & $\succ_{c_{3}}$ & $\succ_{c_{4}}$ & $P_{s_{1}}$ & $P_{s_{1}}^{\prime}$ & $P_{s_{2}}$ & $P_{s_{3}}$ & $P_{s_{4}}$ \\
\hline & $s_{2}$ & $s_{1}$ & $s_{3}$ & $s_{3}$ & $c_{1}$ & $c_{1}$ & $c_{2}$ & $c_{1}$ & $c_{4}$ \\
& $s_{3}$ & $s_{3}$ & $s_{4}$ & $s_{4}$ & $c_{2}$ & $c_{4}$ & $c_{1}$ & $c_{2}$ & $c_{3}$ \\
& $s_{4}$ & $s_{4}$ & $\vdots$ & $\vdots$ & $c_{4}$ & $\vdots$ & $c_{4}$ & $c_{3}$ & $\vdots$ \\
& $s_{1}$ & $s_{2}$ & & & $c_{3}$ & & $c_{3}$ & $c_{4}$ & \\
capacities (ceiling for any type) & 1 & 1 & 1 & 1 & & & & & \\
floor for type $t_{1}$ & 0 & 0 & 0 & 0 & & & & & \\
floor for type $t_{2}$ & 0 & 0 & 1 & 0 & & & & & \\
\hline
\end{tabular}

The precedence list for type $t_{2}$ in DAPL is $r^{t_{2}}: s_{3}, s_{4}$. 
Consider any mechanism $\gamma$ which Pareto dominates DAPL whenever stable improvement is possible. As demonstrated by Example 4, the outcome of DAPL for $P_{S}$ is

$$
\gamma^{D A P L}\left(\mathcal{Z}, \succ_{C}, P_{S}\right)=\left(\begin{array}{cccc}
c_{1} & c_{2} & c_{3} & c_{4} \\
s_{2} & s_{1} & s_{3} & s_{4}
\end{array}\right),
$$

and this is Pareto dominated by $\mu$ in Example 4, which is fair and non-wasteful. Since $\mu$ is the only assignment that Pareto dominates $\gamma^{D A P L}\left(\mathcal{Z}, \succ_{C}, P_{S}\right), \gamma$ must choose $\mu$ for $P_{S}$ :

$$
\gamma\left(\mathcal{Z}, \succ_{C}, P_{S}\right)=\mu=\left(\begin{array}{cccc}
c_{1} & c_{2} & c_{3} & c_{4} \\
s_{1} & s_{2} & s_{3} & s_{4}
\end{array}\right) .
$$

Next, the outcome of DAPL for $\left(P_{s_{1}}^{\prime}, P_{-s_{1}}\right)$ is computed by

$$
\gamma^{D A P L}\left(\mathcal{Z}, \succ_{C}, P_{s_{1}}^{\prime}, P_{-s_{1}}\right)=\left(\begin{array}{cccc}
c_{1} & c_{2} & c_{3} & c_{4} \\
s_{3} & s_{2} & s_{4} & s_{1}
\end{array}\right) \text {. }
$$

And since this is Pareto efficient, we must have $\gamma\left(\mathcal{Z}, \succ_{C}, P_{s_{1}}^{\prime}, P_{-s_{1}}\right)=\gamma^{D A P L}\left(\mathcal{Z}, \succ_{C}, P_{s_{1}}^{\prime}, P_{-s_{1}}\right)$. But now $\gamma_{s_{1}}\left(\mathcal{Z}, \succ_{C}, P_{S}\right) P_{s_{1}}^{\prime} \gamma_{s_{1}}\left(\mathcal{Z}, \succ_{C}, P_{s_{1}}^{\prime}, P_{-s_{1}}\right)$ implies that $\gamma$ is not strategy-proof.

\section{B.5 Proof of Theorem 3}

Take any pair of schools $\left(c, c^{\prime}\right) \in C^{2}$ with $c \neq c^{\prime}$ and any type $t \in T$. Consider any pair of strict rankings $\left(r_{c}^{t}, r_{c^{\prime}}^{t}\right)$ over type- $t$ students which does not have pairwise B-common priority. By definition, the set and/or the order of the bottom $Q_{t}\left(c, c^{\prime}\right)$ students are different between $r_{c}^{t}$ and $r_{c^{\prime}}^{t}$. To be more precise, let $\underline{S}_{t}\left(r^{t}\right) \equiv B_{t}\left(r^{t}, Q_{t}\left(c, c^{\prime}\right)\right)$ be the set of $Q_{t}\left(c, c^{\prime}\right)$ bottom students in a ranking $r^{t}$. Then, at least one of the following two statements must be true: [1] $\underline{S}_{t}\left(r_{c}^{t}\right)=\underline{S}_{t}\left(r_{c^{\prime}}^{t}\right)$ but there are $s, s^{\prime} \in \underline{S}_{t}\left(r_{c}^{t}\right)$ such that $r_{c}^{t}(s)<r_{c}^{t}\left(s^{\prime}\right)$ and $r_{c^{\prime}}^{t}(s)>r_{c^{\prime}}^{t}\left(s^{\prime}\right)$, or $[2] \underline{S}_{t}\left(r_{c}^{t}\right) \neq \underline{S}_{t}\left(r_{c^{\prime}}^{t}\right)$.

Since $Q_{t}\left(c, c^{\prime}\right)>0$, there exists a nonempty $C_{b} \subseteq C \backslash\left\{c, c^{\prime}\right\}$ such that $Q_{t}\left(c, c^{\prime}\right)=$ $\sum_{c^{\prime \prime} \in C_{b}} \underline{q}_{c^{\prime \prime}}^{t}$. Let $C_{n} \equiv C \backslash C_{b}$. As $F\left(C_{b}, t\right) \neq \emptyset$ holds, by Assumption 1, we can find $\mu_{1}^{*}, \mu_{2}^{*} \in$ $F\left(C_{b}, t\right)$ such that (i) there exists $s \in S_{t}$ such that $\mu_{1}^{*}(s)=c$ and $\mu_{2}^{*}(s)=c^{\prime}$; (ii) there is at most one student $s^{\prime} \in S \backslash S_{t}$ such that $\mu_{1}^{*}\left(s^{\prime}\right)=c^{\prime}$ and $\mu_{2}^{*}\left(s^{\prime}\right)=c$; and (iii) for any $s^{\prime \prime} \in S \backslash\left\{s, s^{\prime}\right\}, \mu_{1}^{*}\left(s^{\prime \prime}\right)=\mu_{2}^{*}\left(s^{\prime \prime}\right)$.

[1] $\underline{S}_{t}\left(r_{c}^{t}\right)=\underline{S}_{t}\left(r_{c^{\prime}}^{t}\right)$ but there are $s, s^{\prime} \in \underline{S}_{t}\left(r_{c}^{t}\right)$ such that $r_{c}^{t}(s)<r_{c}^{t}\left(s^{\prime}\right)$ and $r_{c^{\prime}}^{t}(s)>r_{c^{\prime}}^{t}\left(s^{\prime}\right)$.

Let $\bar{S}_{t}=S_{t} \backslash \underline{S}_{t}\left(r_{c}^{t}\right)$. First, we divide $\underline{S}_{t}\left(r_{c}^{t}\right)$ into three subsets: $\underline{S}_{t}^{1}, \underline{S}_{t}^{2}$ and $\underline{S}_{t}^{3}$. $\underline{S}_{t}^{1}$ is the set of students in $\underline{S}_{t}\left(r_{c}^{t}\right)$ whose rank is higher under $r_{c}^{t}$ than under $r_{c^{\prime}}^{t}$. $\underline{S}_{t}^{2}$ is the set of students in $\underline{S}_{t}\left(r_{c}^{t}\right)$ whose rank is lower under $r_{c}^{t}$ than under $r_{c^{\prime}}^{t}$. $\underline{S}_{t}^{3}$ is the set of students 
in $\underline{S}_{t}\left(r_{c}^{t}\right)$ whose rank is the same between under $r_{c}^{t}$ and under $r_{c^{\prime}}^{t}$. More formally, they are defined as

$$
\begin{aligned}
& \underline{S}_{t}^{1} \equiv\left\{\tilde{s} \in \underline{S}_{t}\left(r_{c}^{t}\right)||\left\{\hat{s} \in S_{t} \mid r_{c}^{t}(\tilde{s})<r_{c}^{t}(\hat{s})\right\}|>|\left\{\hat{s} \in S_{t} \mid r_{c}^{t}(\tilde{s})<r_{c}^{t}(\hat{s})\right\} \mid\right\}, \\
& \underline{S}_{t}^{2} \equiv\left\{\tilde{s} \in \underline{S}_{t}\left(r_{c}^{t}\right)||\left\{\hat{s} \in S_{t} \mid r_{c}^{t}(\tilde{s})<r_{c}^{t}(\hat{s})\right\}|<|\left\{\hat{s} \in S_{t} \mid r_{c}^{t}(\tilde{s})<r_{c}^{t}(\hat{s})\right\} \mid\right\}, \\
& \underline{S}_{t}^{3} \equiv\left\{\tilde{s} \in \underline{S}_{t}\left(r_{c}^{t}\right)||\left\{\hat{s} \in S_{t} \mid r_{c}^{t}(\tilde{s})<r_{c}^{t}(\hat{s})\right\}|=|\left\{\hat{s} \in S_{t} \mid r_{c}^{t}(\tilde{s})<r_{c}^{t}(\hat{s})\right\} \mid\right\} .
\end{aligned}
$$

Note that we have $\underline{S}_{t}^{1} \neq \emptyset$ and $\underline{S}_{t}^{2} \neq \emptyset$.

Now consider the following preferences of students. Let every student in $\underline{S}_{t}^{1}$ prefer $c^{\prime}$ most, $c$ next, schools in $C_{n} \backslash\left\{c, c^{\prime}\right\}$ next. Let every student in $\underline{S}_{t}^{2}$ prefer $c$ most, $c^{\prime}$ next, and schools in $C_{n} \backslash\left\{c, c^{\prime}\right\}$ next. Let every student in $\underline{S}_{t}^{3}$ prefer schools in $C_{b}$ most, $c$ and $c^{\prime}$ next, and schools in $C_{n} \backslash\left\{c, c^{\prime}\right\}$ next. Let $s^{\prime \prime}$ be the student such that $r_{c}^{t}\left(s^{\prime \prime}\right)<r_{c}^{t}(\tilde{s})$ for any $\tilde{s} \in S_{t} \backslash\left\{s^{\prime \prime}\right\}$. Note that $s^{\prime \prime} \in \bar{S}_{t}{ }^{23}$ Let $s^{\prime \prime}$ prefer some school in $C_{b}$ most. Because $\left|\underline{S}_{t}\left(r_{c}^{t}\right)\right|=Q_{t}\left(c, c^{\prime}\right)$ and $\underline{S}_{t}^{1}, \underline{S}_{t}^{2} \neq \emptyset$, we can pick preferences of students in $\underline{S}_{t}^{3} \cup\left\{s^{\prime \prime}\right\}$ so that by assigning every student in $\underline{S}_{t}^{3} \cup\left\{s^{\prime \prime}\right\}$ to her first choice in $C_{b}$, we can still achieve the distribution of each type of students under $\mu_{1}^{*}$ and $\mu_{2}^{*}$. Finally, let all other students in $\bar{S}_{t} \backslash\left\{s^{\prime \prime}\right\}$ prefer schools in $C_{n}$. Since $\mu_{1}^{*}, \mu_{2}^{*} \in F\left(C_{b}, t\right)$, we can pick preferences of students in $\bar{S}_{t} \backslash\left\{s^{\prime \prime}\right\}$ so that every student in $\bar{S}_{t} \backslash\left\{s^{\prime \prime}\right\}$ is assigned to her first choice and we can still achieve the distribution of each type of students under $\mu_{1}^{*}$ and $\mu_{2}^{*}$.

The following table summarizes the preferences of students. (Information about the exact order of schools is omitted.)

\begin{tabular}{ccccc}
$P_{\underline{S}_{t}^{1}}$ & $P_{\underline{S}_{t}^{2}}$ & $P_{\underline{S}_{t}^{3}}$ & $P_{s^{\prime \prime}}$ & $P_{\bar{S}_{t} \backslash\left\{s^{\prime \prime}\right\}}$ \\
\hline$c^{\prime}$ & $c$ & $C_{b}$ & $C_{b}$ & $C_{n}$ \\
$c$ & $c^{\prime}$ & $\left\{c, c^{\prime}\right\}$ & $\vdots$ & $\vdots$ \\
$C_{n} \backslash\left\{c, c^{\prime}\right\}$ & $C_{n} \backslash\left\{c, c^{\prime}\right\}$ & $C_{n} \backslash\left\{c, c^{\prime}\right\}$ & & \\
$\vdots$ & $\vdots$ & & &
\end{tabular}

Let us construct $\succ_{C}$ which satisfies the following conditions: (i) it is consistent with $\left(r_{c}^{t}, r_{c^{\prime}}^{t}\right)$, (ii) $\left(\succ_{c}, \succ_{c^{\prime \prime}}\right)$ has a common priority order for type $t$ for any $c^{\prime \prime} \in C \backslash\left\{c, c^{\prime}\right\}$ and (iii) $\left(\succ_{\tilde{c}}, \succ_{\hat{c}}\right)$ has a common priority order for any other type $t^{\prime} \in T \backslash\{t\}$ and for any pair of schools $(\tilde{c}, \hat{c}) \in C^{2}$. We can find such $\succ_{C}$ by prioritizing any student in $S \backslash\left\{S_{t}\right\}$ below type- $t$ students by some common order across schools. The next table summarizes this idea:

\footnotetext{
${ }^{23}$ This is because if $s^{\prime \prime} \in \underline{S}_{t}\left(r_{c}^{t}\right)$, this means that $\underline{S}_{t}\left(r_{c}^{t}\right)=S_{t}$ and hence $\sum_{c \in C} \underline{q}_{c}^{t}=\left|S_{t}\right|$, which is precluded in our model.
} 


\begin{tabular}{|c|c|c|c|c|}
\hline & $\succ_{C_{n} \backslash\left\{c, c^{\prime}\right\}}$ & $\succ_{c}$ & $\succ_{c^{\prime}}$ & $\succ_{C_{b}}$ \\
\hline & $\bar{S}_{t}$ & $\bar{S}_{t}$ & $\bar{S}_{t}$ & $\bar{S}_{t}$ \\
\hline & $\vdots$ & $\vdots$ & $\vdots$ & $\vdots$ \\
\hline & $s$ & $s$ & $s^{\prime}$ & $s$ \\
\hline & : & $\vdots$ & $\vdots$ & $\vdots$ \\
\hline & $s^{\prime}$ & $s^{\prime}$ & $s$ & $s^{\prime}$ \\
\hline & $\vdots$ & $\vdots$ & $\vdots$ & $\vdots$ \\
\hline & $S \backslash S_{t}$ & $S \backslash S_{t}$ & $S \backslash S_{t}$ & $\begin{array}{c}S \backslash S_{t} \\
Q_{t}\left(c, c^{\prime}\right)\end{array}$ \\
\hline
\end{tabular}

Now consider feasible assignments that are fair an non-wasteful. First, it is easy to see that in any fair an non-wasteful assignment, $s^{\prime \prime}$ must be assigned to her first choice in $C_{b}$ because $s^{\prime \prime}$ has the highest priority in that school. All students in $\bar{S}_{t} \backslash\left\{s^{\prime \prime}\right\}$ must be assigned to their first choices because students in $\bar{S}_{t}$ have higher priority than those in $\underline{S}_{t}\left(r_{c}^{t}\right)$ at any school.

Next, consider students in $\underline{S}_{t}\left(r_{c}^{t}\right)$. If some student in $\underline{S}_{t}\left(r_{c}^{t}\right)$ is assigned to schools in $C_{n}$, the number of such students is at most one because there are $Q_{t}\left(c, c^{\prime}\right)-1$ unfilled minimum quotas of schools in $C_{b}$. Now, all students in $\underline{S}_{t}^{3}$ must be assigned to their first choices in $C_{b}$. This is because if a student $\tilde{s} \in \underline{S}_{t}^{3}$ is assigned to some school in $C_{n}$, then one of the following three scenarios must be true: (i) $\tilde{s}$ would justifiably claim an empty slot or justifiably envy a student of a different type at $c$ or $c^{\prime}$ if $\tilde{s}$ is assigned to $C_{n} \backslash\left\{c, c^{\prime}\right\}$, (ii) $\tilde{s}$ would justifiably envy another student in $\underline{S}_{t}\left(r_{c}^{t}\right)$ assigned to a school in $C_{b}$, or (iii) some student in $\underline{S}_{t}\left(r_{c}^{t}\right)$ would justifiably envy $\tilde{s}$ if $\tilde{s}$ is assigned to $c$ or $c^{\prime}$. Thus, one student who can be assigned to a school in $C_{n}$ under a fair and non-wasteful assignment should be in $\underline{S}_{t}^{1} \cup \underline{S}_{t}^{2}$. Note that by assigning students in $\bar{S}_{t} \backslash\left\{s^{\prime \prime}\right\}$ to their first choices, the distribution of each type of students under $\mu_{1}^{*}$ and $\mu_{2}^{*}$ can be achieved. Since $\mu_{1}^{*}$ and $\mu_{2}^{*}$ are both feasible, one student in $\underline{S}_{t}^{1} \cup \underline{S}_{t}^{2}$ can be assigned to either $c$ or $c^{\prime}$.

Then let us consider the available slots of $c$ and $c^{\prime}$ (one for each) and the $Q_{t}\left(c, c^{\prime}\right)-1$ unfilled minimum quotas of schools in $C_{b}$. Let $s_{1}$ be any student in $\underline{S}_{t}^{1}$ and $s_{2}$ be any student in $\underline{S}_{t}^{2}$. Since the minimum quotas must be satisfied, there are four possibilities:

$$
\begin{aligned}
& \mu_{1}=\left(\begin{array}{ccc}
c & c^{\prime} & C_{b} \\
s_{1} & & \underline{S}_{t}\left(r_{c}^{t}\right) \backslash\left\{s_{1}\right\}
\end{array}\right), \mu_{2}=\left(\begin{array}{ccc}
c & c^{\prime} & C_{b} \\
& s_{1} & \underline{S}_{t}\left(r_{c}^{t}\right) \backslash\left\{s_{1}\right\}
\end{array}\right), \\
& \mu_{3}=\left(\begin{array}{ccc}
c & c^{\prime} & C_{b} \\
& s_{2} & \underline{S}_{t}\left(r_{c}^{t}\right) \backslash\left\{s_{2}\right\}
\end{array}\right), \mu_{4}=\left(\begin{array}{ccc}
c & c^{\prime} & C_{b} \\
s_{2} & & \underline{S}_{t}\left(r_{c}^{t}\right) \backslash\left\{s_{2}\right\}
\end{array}\right) .
\end{aligned}
$$


Now this has the same structure as the counterexample of Theorem 1.

1. $\mu_{1}$ is not fair or is wasteful as $s_{1}$ justifiably envies a student of a different type or justifiably claims an empty slot at $c^{\prime}$,

2. $\mu_{2}$ is not fair as there must be a student in $\underline{S}_{t}^{2}$ who justifiably envies $s_{1}$ at $c^{\prime}$,

3. $\mu_{3}$ is not fair or is wasteful as $s_{2}$ justifiably envies a student of a different type or justifiably claims an empty slot at $c$, and

4. $\mu_{4}$ is not fair as there must be a student in $\underline{S}_{t}^{1}$ who justifiably envies $s_{2}$ at $c$.

Therefore, there exists no feasible assignment that is fair and wasteful.

$[2] \underline{S}_{t}\left(r_{c}^{t}\right) \neq \underline{S}_{t}\left(r_{c^{\prime}}^{t}\right)$.

First, we divide $S_{t}$ into four subsets: $S_{t}^{1}, S_{t}^{2}, S_{t}^{3}$ and $S_{t}^{4}$, which are defined as follows:

$$
\begin{aligned}
S_{t}^{1} & \equiv \underline{S}_{t}\left(r_{c^{\prime}}^{t}\right) \backslash \underline{S}_{t}\left(r_{c}^{t}\right), \\
S_{t}^{2} & \equiv \underline{S}_{t}\left(r_{c}^{t}\right) \backslash \underline{S}_{t}\left(r_{c^{\prime}}^{t}\right), \\
S_{t}^{3} & \equiv \underline{S}_{t}\left(r_{c}^{t}\right) \cap \underline{S}_{t}\left(r_{c^{\prime}}^{t}\right), \\
S_{t}^{4} & \equiv S_{t} \backslash\left(\underline{S}_{t}\left(r_{c}^{t}\right) \cup \underline{S}_{t}\left(r_{c^{\prime}}^{t}\right)\right) .
\end{aligned}
$$

Note that we have $S_{t}^{1} \neq \emptyset, S_{t}^{2} \neq \emptyset$ and $\left|S_{t}^{3}\right|<Q_{t}\left(c, c^{\prime}\right)$.

Now consider the following preferences of students. Let every student in $S_{t}^{1}$ prefer $c^{\prime}$ most, $c$ next, schools in $C_{n} \backslash\left\{c, c^{\prime}\right\}$ next. Let every student in $S_{t}^{2}$ prefer $c$ most, $c^{\prime}$ next, and schools in $C_{n} \backslash\left\{c, c^{\prime}\right\}$ next. Let every student in $S_{t}^{3}$ prefer schools in $C_{b}$ most, $c$ and $c^{\prime}$ next, and schools in $C_{n} \backslash\left\{c, c^{\prime}\right\}$ next. Because $\left|\underline{S}_{t}\left(r_{c}^{t}\right)\right|=\left|\underline{S}_{t}\left(r_{c^{\prime}}^{t}\right)\right|=Q_{t}\left(c, c^{\prime}\right)$ and $S_{t}^{1}, S_{t}^{2} \neq \emptyset$, we can pick preferences of students in $S_{t}^{3}$ so that by assigning every student in $S_{t}^{3}$ to her first choice in $C_{b}$, we can achieve the distributions of each type of students that are the same as either of $\mu_{1}^{*}$ and $\mu_{2}^{*}$. Finally, let all other students in $S_{t}^{4}$ prefer schools in $C_{n}$. Since $\mu_{1}^{*}, \mu_{2}^{*} \in F\left(C_{b}, t\right)$, we can pick preferences of students in $S_{t}^{4}$ so that every student in $S_{t}^{4}$ is assigned to her first choice and we can still achieve the distribution of each type of students under $\mu_{1}^{*}$ and $\mu_{2}^{*}$.

The following table summarizes the preferences of students. (Information about the exact order of schools is omitted.)

\begin{tabular}{cccc}
$P_{S_{t}^{1}}$ & $P_{S_{t}^{2}}$ & $P_{S_{t}^{3}}$ & $P_{S_{t}^{4}}$ \\
\hline$c^{\prime}$ & $c$ & $C_{b}$ & $C_{n}$ \\
$c$ & $c^{\prime}$ & $\left\{c, c^{\prime}\right\}$ & $\vdots$ \\
$C_{n} \backslash\left\{c, c^{\prime}\right\}$ & $C_{n} \backslash\left\{c, c^{\prime}\right\}$ & $\vdots$ & \\
$\vdots$ & $\vdots$ & &
\end{tabular}


As in the first case, we can find an appropriate $\succ_{C}$ by prioritizing any student in $S \backslash\left\{S_{t}\right\}$ below type- $t$ students by some common order across schools. The next table summarizes this idea:

\begin{tabular}{ccccc} 
& $\succ_{C_{n} \backslash\left\{c, c^{\prime}\right\}}$ & $\succ_{c}$ & $\succ_{c^{\prime}}$ & $\succ_{C_{b}}$ \\
\hline & $S_{t}^{4} \cup S_{t}^{1}$ & $S_{t}^{4} \cup S_{t}^{1}$ & $S_{t}^{4} \cup S_{t}^{2}$ & $S_{t}^{4} \cup S_{t}^{1}$ \\
& $S_{t}^{3} \cup S_{t}^{2}$ & $S_{t}^{3} \cup S_{t}^{2}$ & $S_{t}^{3} \cup S_{t}^{1}$ & $S_{t}^{3} \cup S_{t}^{2}$ \\
& $S \backslash S_{t}$ & $S \backslash S_{t}$ & $S \backslash S_{t}$ & $S \backslash S_{t}$ \\
floor for type $t$ & & & & $Q_{t}\left(c, c^{\prime}\right)$ \\
\hline
\end{tabular}

Now consider feasible assignments that are fair an non-wasteful. First, all students in $S_{t}^{4}$ must be assigned to their first choices because otherwise students in $S_{t}^{4}$ would justifiably envy some student assigned to a school in $C_{n}$. Next, students in $S_{t}^{3}$ must be all assigned to their first choices in $C_{b}$. This is because if a student $\tilde{s} \in S_{t}^{3}$ is assigned to some school in $C_{n}$, then one of the following three scenarios must be true: (i) $\tilde{s}$ would justifiably claim an empty slot or justifiably envy a student of a different type at $c$ or $c^{\prime}$ if $\tilde{s}$ is assigned to $C_{n} \backslash\left\{c, c^{\prime}\right\}$, (ii) $\tilde{s}$ would justifiably envy another student in $\underline{S}_{t}\left(r_{c}^{t}\right)$ assigned to a school in $C_{b}$, or (iii) some student in $\underline{S}_{t}\left(r_{c}^{t}\right)$ would justifiably envy $\tilde{s}$ if $\tilde{s}$ is assigned to $c$ or $c^{\prime}$. Thus, under any feasible, fair and non-wasteful assignment, $Q_{t}\left(c, c^{\prime}\right)-\left|S_{t}^{3}\right|$ students in $S_{t}^{1} \cup S_{t}^{2}$ must fill the minimum quotas of schools in $C_{b}$, and other students in $S_{t}^{1} \cup S_{t}^{2}$ can be assigned to schools in $C_{n}$.

There are three possibilities.

1. When the $Q_{t}\left(c, c^{\prime}\right)-\left|S_{t}^{3}\right|$ minimum quotas of schools in $C_{b}$ are all filled by students in $S_{t}^{1}$, all students in $S_{t}^{2}$ are assigned to $c$ or $c^{\prime}$. Because the distributions of both $\mu_{1}^{*}$ and $\mu_{2}^{*}$ can be achieved, at least one student $s_{2} \in S_{t}^{2}$ must be assigned to $c$ because otherwise $s_{2}$ would justifiably claim an empty slot or envy another student of a different type at $c$. But then, some student in $S_{t}^{1}$ justifiably envies $s_{2}$.

2. When the $Q_{t}\left(c, c^{\prime}\right)-\left|S_{t}^{3}\right|$ minimum quotas of schools in $C_{b}$ are all filled by students in $S_{t}^{2}$, all students in $S_{t}^{1}$ are assigned to $c$ or $c^{\prime}$. Because the distributions of both $\mu_{1}^{*}$ and $\mu_{2}^{*}$ can be achieved, at least one student $s_{1} \in S_{t}^{1}$ must be assigned to $c^{\prime}$ because otherwise $s_{1}$ would justifiably claim an empty slot or envy another student of a different type at $c^{\prime}$. But then, some student in $S_{t}^{2}$ justifiably envies $s_{1}$.

3. When the $Q_{t}\left(c, c^{\prime}\right)-\left|S_{t}^{3}\right|$ minimum quotas of schools in $C_{b}$ are filled by students in both $S_{t}^{1}$ and $S_{t}^{2}$, the rest of students in $S_{t}^{1}$ and $S_{t}^{2}$ are assigned to $c$ or $c^{\prime}$. Because the distributions of both $\mu_{1}^{*}$ and $\mu_{2}^{*}$ can be achieved, at least one student $s_{1} \in S_{t}^{1}$ must be assigned to $c^{\prime}$ or one student $s_{2} \in S_{t}^{2}$ must be assigned to $c$ because otherwise there 
is at least one student in $S_{t}^{1} \cup S_{t}^{2}$ who would justifiably claim an empty slot or envy another student of a different type. But then, some student in $S_{t}^{2}$ who is assigned to a school in $C_{b}$ justifiably envies $s_{1}$, or some student in $S_{t}^{1}$ who is assigned to a school in $C_{b}$ justifiably envies $s_{2}$.

Therefore, there exists no feasible assignment that is fair and wasteful.

\section{Appendix C Algorithm for Computing $Q_{t}\left(c, c^{\prime}\right)$}

Take any pair of schools $\left(c, c^{\prime}\right) \in C^{2}$ with $c \neq c^{\prime}$ and any type $t \in T$. Label the sets in $2^{C \backslash\left\{c, c^{\prime}\right\}}$ as $\tilde{C}_{1}, \tilde{C}_{2}, \ldots$ to satisfy $\sum_{c^{\prime \prime} \in \tilde{C}_{l}} \underline{q}_{c^{\prime \prime}}^{t} \geq \sum_{c^{\prime \prime} \in \tilde{C}_{l+1}} \underline{q}_{c^{\prime \prime}}^{t}$ for each $l \in\left\{1, \ldots, 2^{m-2}-1\right\}$.

In the algorithm, we examine these sets in the order of $\tilde{C}_{1}, \tilde{C}_{2}, \ldots$ until we find $\tilde{C}_{l}$ with $F\left(\tilde{C}_{l}, t\right) \neq \emptyset{ }^{24}$ The input is a primitive problem $\mathcal{Z}$. Each step $l$ runs as follows:

1. For each $c^{\prime \prime} \in \tilde{C}_{l}$, reduce its type- $t$ maximum quota to its type- $t$ minimum quota, i.e., define a new type-t maximum quota $\hat{\bar{q}}_{c^{\prime \prime}}^{t} \equiv \underline{q}_{c^{\prime \prime}}^{t}$.

2. Consider a new primitive problem $\hat{\mathcal{Z}}$ where the type- $t$ maximum quota for each $c^{\prime \prime} \in \tilde{C}_{l}$ is $\hat{\bar{q}}_{c^{\prime \prime}}^{t}$ and other parameters are the same as $\mathcal{Z}$. As discussed in Appendix A of Ehlers et al. (2014), since the constraints correspond to the transportation problem in the operations research literature, we can detect whether there is a feasible assignment in $\hat{\mathcal{Z}}$ in a polynomial time.

3. If there is a feasible assignment in $\hat{\mathcal{Z}}$, then this means $F\left(\tilde{C}_{l}, t\right) \neq \emptyset$ and $Q_{t}\left(c, c^{\prime}\right)=$ $\sum_{c^{\prime \prime} \in \tilde{C}_{l}} \underline{q}_{c^{\prime \prime}}^{t}$. The algorithm terminates in this case. If not, proceed to step $l+1$.

\section{Appendix D Omitted Examples}

\section{Appendix D.1 DAPL without Additional Rejection Steps}

The following example illustrates that DAPL without Additional Rejection Steps may not be fair for problems with B-common priority.

\footnotetext{
${ }^{24}$ This means that the maximum number of steps is $2^{m-2}-1$. However, we can significantly reduce the number of steps if multiple schools have exactly the same constraints. Moreover, if the maximum quotas are not so tight, it is likely that the algorithm stops in early steps.
} 
Example 6. Consider the following problem: $C=\left\{c_{1}, c_{2}, c_{3}\right\}, S=\left\{s_{1}, s_{2}, s_{3}, s_{4}, s_{5}\right\}$ and $T=\left\{t_{1}\right\}$. All students are of the same type. Priorities, preferences, capacities and typespecific constraints are summarized in the table below.

\begin{tabular}{ccccccccc}
$\succ_{c_{1}}$ & $\succ_{c_{2}}$ & $\succ_{c_{3}}$ & $P_{s_{1}}$ & $P_{s_{2}}$ & $P_{s_{2}}$ & $P_{s_{4}}$ & $P_{s_{5}}$ \\
\hline$s_{4}$ & $s_{1}$ & $s_{1}$ & $c_{1}$ & $c_{1}$ & $c_{1}$ & $c_{1}$ & $\vdots$ \\
$s_{1}$ & $s_{2}$ & $s_{2}$ & $c_{2}$ & $c_{2}$ & $c_{2}$ & $c_{2}$ & \\
$s_{2}$ & $s_{3}$ & $s_{3}$ & $\vdots$ & $\vdots$ & $\vdots$ & $\vdots$ & \\
$s_{3}$ & $s_{4}$ & $s_{4}$ & & & & & \\
$s_{5}$ & $s_{5}$ & $s_{5}$ & & & & &
\end{tabular}

capacity (ceiling for type $\left.t_{1}\right) \quad 5 \quad 5 \quad 5$

floor for type $t_{1} \quad 2 \quad 1 \quad 1$

$\succ_{C}$ has B-common priority because $Q_{t_{1}}\left(c_{2}, c_{3}\right)=2$ and $Q_{t_{1}}\left(c_{1}, c_{2}\right)=Q_{t_{1}}\left(c_{1}, c_{3}\right)=1$. The precedence list $r^{t_{1}}$ of DAPL must satisfy $r^{t_{1}}\left(s_{4}\right)=4$ and $r^{t_{1}}\left(s_{5}\right)=5$ because $Q_{t_{1}}=$ $Q_{t_{1}}\left(c_{2}, c_{3}\right)=2$.

First, DAPL without Additional Rejection Steps achieves the following assignment:

$$
\mu^{D A P L w A R S}=\left(\begin{array}{ccc}
c_{1} & c_{2} & c_{3} \\
\left\{s_{1}, s_{2}, s_{3}\right\} & s_{4} & s_{5}
\end{array}\right) .
$$

This is because when $s_{1}, s_{2}$, and $s_{3}$ are accepted to $c_{1}$, two artificial students fill the minimum quotas of $c_{2}$ and $c_{3}$, and the DA stage terminates. However, $s_{4}$ justifiably envies $s_{1}, s_{2}$, and $s_{3}$ at $c_{1}$ and $\mu^{D A P L w A R S}$ is not fair.

On the other hand, under DAPL, there is an additional rejection step because when $s_{1}$, $s_{2}$, and $s_{3}$ are accepted to $c_{1}, c_{1}$ is the only school whose type- $t$ division is filled by real students. Then, one artificial student is rejected from $c_{3}^{t_{1}}$, and in the next step, $s_{4}$ proposes to $c_{1} . c_{1}$ accepts $s_{4}$ instead of $s_{3}$, and $s_{3}$ is finally assigned to $c_{2}$. To summarize, the outcome of DAPL is

$$
\mu^{D A P L}=\left(\begin{array}{ccc}
c_{1} & c_{2} & c_{3} \\
\left\{s_{1}, s_{2}, s_{4}\right\} & s_{3} & s_{5}
\end{array}\right),
$$

and this is a fair assignment.

\section{Appendix D.2 Serial Dictatorship}

Here, we discuss the properties of the serial dictatorship (SD) mechanism suitably defined for our problem. 


\section{Serial Dictatorship}

Fix an arbitrary precedence list $r$ over all students.

In each step $l \in\{1, \ldots,|S|\}$, student $s \in S$ with $r(s)=l$ chooses her most preferred school among schools $c$ with the following condition: there is $\mu \in F$ in which (i) all students $s^{\prime} \in S$ with $r\left(s^{\prime}\right) \in\{1, \ldots, l-1\}$ are assigned to their chosen schools in the previous steps, and (ii) $\mu(s)=c$.

By $F \neq \emptyset$, each student can choose at least one school, and the SD terminates after step $|S|$.

SD is clearly feasible, non-wasteful and group strategy-proof. However, it violates strict PL-fairness in two ways: (i) SD is not strictly PL-fair for same types, and (ii) it is not fair across different types. The following example demonstrates these two points.

Observation 4. SD (with any order of students) is not strictly PL-fair for same types.

Example 7. Consider the following problem: $C=\left\{c_{1}, c_{2}\right\}, S=\left\{s_{1}, s_{2}\right\}$ and $T=\left\{t_{1}\right\}$. Priorities, capacities and type-specific constraints are summarized in the table below.

\begin{tabular}{ccc} 
& $\succ_{c_{1}}$ & $\succ_{c_{2}}$ \\
\hline & $s_{1}$ & $s_{2}$ \\
& $s_{2}$ & $s_{1}$ \\
capacity (ceiling for type $\left.t_{1}\right)$ & 1 & 1 \\
floor for type $t_{1}$ & 0 & 0 \\
\hline
\end{tabular}

Note that $Q_{t_{1}}=0$ in this problem.

For SD with $r: s_{1}, s_{2}$, consider preferences $P_{S}$ such that $c_{2} P_{s} c_{1}$ for all $s \in S$. Then the outcome is

$$
\mu=\left(\begin{array}{ll}
c_{1} & c_{2} \\
s_{2} & s_{1}
\end{array}\right),
$$

but $s_{2}$ PL-envies $s_{1}$ at $c_{2}$. Thus, this is not strictly PL-fair.

For SD with $r: s_{2}, s_{1}$, consider preferences $P_{S}^{\prime}$ such that $c_{1} P_{s}^{\prime} c_{2}$ for all $s \in S$. Then the outcome is $\mu$. But this is not strictly PL-fair because $s_{1}$ PL-envies $s_{2}$ at $c_{1}$.

To see that SD is not fair across different types, we can slightly change Example 7 in the following way: $T=\left\{t_{1}, t_{2}\right\}, \tau\left(s_{1}\right)=t_{1}, \tau\left(s_{2}\right)=t_{2}, q_{c_{1}}=q_{c_{2}}=1$, and $\left(\bar{q}_{c}^{t}, q_{c}^{t}\right)=(1,0)$ for each $(t, c) \in T \times C$. Then, the same logic as Example 7 applies and SD with either order of students fails to be fair across different types.

Observation 5. SD (with any order of students) is not fair across different types. 


\section{Appendix D.3 Stable Assignments without B-common Priority}

The following example shows that there are priority rankings over all students for which the B-common priority condition is violated but a stable assignment exists for any preferences.

Example 8. Consider the following problem: $C=\left\{c_{1}, c_{2}, c_{3}\right\}, S=\left\{s_{1}, s_{2}, s_{3}, s_{4}\right\}, T=$ $\left\{t_{1}, t_{2}\right\}, \tau\left(s_{1}\right)=\tau\left(s_{2}\right)=t_{1}$, and $\tau\left(s_{3}\right)=\tau\left(s_{4}\right)=t_{2}$. Priorities, capacities and type-specific constraints are summarized in the table below.

\begin{tabular}{cccc} 
& $\succ_{c_{1}}$ & $\succ_{c_{2}}$ & $\succ_{c_{3}}$ \\
\hline & $s_{3}$ & $s_{4}$ & $s_{1}$ \\
& $s_{4}$ & $s_{3}$ & $s_{2}$ \\
& $s_{1}$ & $s_{2}$ & $\vdots$ \\
& $s_{2}$ & $s_{1}$ & \\
capacities (ceiling for any type) & 1 & 1 & 2 \\
floor for type $t_{1}$ & 0 & 0 & 1 \\
floor for type $t_{2}$ & 0 & 0 & 0 \\
\hline
\end{tabular}

Here, B-common priority is violated because $c_{1}$ and $c_{2}$ rank $s_{1}$ and $s_{2}$ differently but we have $Q_{t_{1}}\left(c_{1}\right)=Q_{t_{1}}\left(c_{2}\right)=Q_{t_{1}}\left(c_{1}, c_{2}\right)=1$.

Consider the following feasible assignment:

$$
\mu=\left(\begin{array}{ccc}
c_{1} & c_{2} & c_{3} \\
s_{3} & s_{4} & \left\{s_{1}, s_{2}\right\}
\end{array}\right)
$$

Since no student can justifiably envy another student or justifiably claim an empty slot, $\mu$ is fair and non-wasteful for any preferences. 